\title{
2-Oxo-2-polyfluoroalkylethane-1-sulfones and -sulfamides in the three-component reactions leading to pyrimidine derivatives
}

\author{
Vadim M. Timoshenko,* Yuriy M. Markitanov, Yuriy O. Salimov, \\ and Yuriy G. Shermolovich \\ Institute of Organic Chemistry, NAS of Ukraine, Murmanska str. 5, Kyiv, 02660, Ukraine \\ E-mail:vadim@ioch.kiev.ua
}

DOI: $\underline{\text { http://dx.doi.org/10.3998/ark.5550190.p008.582 }}$

\begin{abstract}
2-Oxo-2-polyfluoroalkylethane-1-sulfones and -sulfamides undergo Biginelli reaction with aryl aldehydes and (thio)urea to give 4-hydroxy-4-polyfluoroalkyl-5-sulfonyl-6-aryltetrahydropyrimidin-2(1H)-(thi)ones, whereas three-component reaction with urea and trialkyl orthoformate provides 4-hydroxy-4-polyfluoroalkyl-5-sulfonyl-3,4-dihydropyrimidin-2(1H)ones. Some chemical properties of the synthesized pyrimidinones are discussed.
\end{abstract}

Keywords: Multicomponent reaction, Biginelli reaction, pyrimidinone, polyfluoroalkyl, ketosulfone, ketosulfamide

\section{Introduction}

Multicomponent reactions allow the construction of complex compounds from several simple functional substrates in a one-pot procedure ${ }^{1,2}$ and due to exceptional synthetic efficiency in comparison with traditional multistep approach attract considerable interest of synthetic chemists. Of particular interest are multicomponent reactions forming heterocyclic compounds (drug-like scaffolds) representing potential targets for medicinal chemistry. Among a number of pharmaceutically important heterocycles pyrimidinones ${ }^{3-5}$ and structurally close compounds as well as their fluoroalkyl derivatives ${ }^{6-9}$ are also known to exert bioactivities which stimulates the invention of simple approaches for their synthesis. One of the most effective strategies for the construction of pyrimidinone scaffold is the broadly studied Biginelli reaction. ${ }^{10-12}$ In its traditional form three-component Biginelli cyclocondensation involves a combination of $\beta$ ketoester and aromatic aldehyde with urea in protic solvent under acidic conditions resulting in the formation of 3,4-dihydropyrimidin-2(1H)-ones known also as classical "Biginelli compounds". Throughout the exploration of the Biginelli reaction potential, by diversifying all three components, the scope of the employed $\beta$-dicarbonyl substrates has been extended by their 
analogues containing functional groups or heterofunction, which considerably enhanced the versatility of the 3,4-dihydropyrimidin-2(1H)-one backbone. ${ }^{2,3}$ During the recent decades various building blocks combinations adjusted to differing reaction conditions and catalytic systems have been thoroughly investigated and numerous improved methodologies and modifications of the classical Biginelli reaction have emerged. Still, elaboration of convenient one-pot task-oriented approaches for the generation of novel pyrimidine scaffolds beyond the classical Biginelli reaction remains an actual challenge of organic synthesis, which was the purpose of our work.

Herein we report on the applicability of 2-oxo-2-polyfluoroalkylethane-1-sulfones and -sulfamides as substrates of the three-component reactions for the preparation of polyfluoroalkyl pyrimidine derivatives substituted with a sulfonyl or sulfamide moiety as well as on the investigation of some chemical properties of the products obtained.

\section{Results and Discussion}

Literature data indicate that attempts to subject $\beta$-ketosulfones as $\beta$-ketoester alternatives to the Biginelli reaction for the synthesis of sulfonyl-substituted pyrimidinones were fruitless. Microwave-assisted multicomponent reaction of methylsulfonyl acetone, aromatic aldehydes and urea yielded unusual Hanztsch dihydropyridines. ${ }^{13} \beta$-Ketosulfone containing perhaloalkyl substituent $\left(\mathrm{R}=\left(\mathrm{CF}_{2}\right)_{4} \mathrm{Cl}\right)$ at sulfone moiety by reacting with benzaldehyde and urea under the classical Biginelli procedure afforded 2,3-dihydrofuran instead of the expected dihydropyrimidinone. ${ }^{14}$ Biginelli-type cyclocondensation reaction of benzaldehyde, urea and tetrahydrothiopyran-3-one $S, S$-dioxide - cyclic $\beta$-ketosulfone, which was used as alternative carbonyl substrate gave fused-ring pyrimidine, while the acyclic version of this protocol with phenylsulfonyl acetophenone to provide a pyrimidinone scaffold failed. ${ }^{15}$ Recently ${ }^{16}$ we have shown that, as distinct from the mentioned literature cases, $\beta$-polufluoroalkyl- $\beta$-keto-sulfones $\mathbf{1 a}, \mathbf{b}$ as well as -sulfamides 1c,d are able to undergo a three-component Biginelli reaction with aryl aldehydes and urea to give the desired sulfonyl-substituted tetrahydropyrimidin-2(1H)-ones (compounds 2a-e,m-p) (Table 1). In this paper we have broaden the scope of the application of fluorinated keto compounds $\mathbf{1}$ in multicomponent reactions for the synthesis of pyrimidine derivatives as well as studied the structural features of the obtained Biginelli tetrahydropyrimidinones. 
Table 1. Biginelli reaction of 2-oxo-2-polyfluoroalkylethane-1-sulfones 1a,b and -sulfamides 1c,d<smiles>O=C[Al]</smiles><smiles>[R6][R6]#[R16]</smiles>

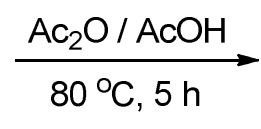<smiles>[X]C1=NC([Al])C(O)C([R])(O)N1</smiles>

\begin{tabular}{|c|c|c|c|c|c|c|}
\hline Ketone & $\mathrm{R}_{\mathrm{F}}$ & $\mathrm{R}$ & $\mathrm{Ar}$ & $X$ & Product & Yield $(\%)^{\mathrm{a}}$ \\
\hline $1 \mathbf{a}$ & $\mathrm{CF}_{3}$ & $p$-Tol & $\mathrm{Ph}$ & $\mathrm{O}$ & $\mathbf{2 a}$ & 85 \\
\hline $\mathbf{1 a}$ & $\mathrm{CF}_{3}$ & $p$-Tol & $p-\mathrm{MeOC}_{6} \mathrm{H}_{4}$ & $\mathrm{O}$ & $2 b$ & 83 \\
\hline $1 \mathbf{a}$ & $\mathrm{CF}_{3}$ & $p$-Tol & $p-\mathrm{BrC}_{6} \mathrm{H}_{4}$ & $\mathrm{O}$ & $2 c$ & 77 \\
\hline $1 b$ & $\mathrm{HCF}_{2} \mathrm{CF}_{2}$ & $p$-Tol & $\mathrm{Ph}$ & $\mathrm{O}$ & $2 d$ & 80 \\
\hline $1 b$ & $\mathrm{HCF}_{2} \mathrm{CF}_{2}$ & $p$-Tol & $p-\mathrm{MeOC}_{6} \mathrm{H}_{4}$ & $\mathrm{O}$ & $2 e$ & 75 \\
\hline $1 b$ & $\mathrm{HCF}_{2} \mathrm{CF}_{2}$ & $p$-Tol & $p-\mathrm{BrC}_{6} \mathrm{H}_{4}$ & $\mathrm{O}$ & $2 f$ & 66 \\
\hline $1 \mathbf{a}$ & $\mathrm{CF}_{3}$ & $p$-Tol & $\mathrm{Ph}$ & S & $2 \mathrm{~g}$ & 56 \\
\hline $1 \mathbf{a}$ & $\mathrm{CF}_{3}$ & $p$-Tol & $p-\mathrm{MeOC}_{6} \mathrm{H}_{4}$ & $S$ & $2 \mathrm{~h}$ & 60 \\
\hline $1 \mathbf{a}$ & $\mathrm{CF}_{3}$ & $p$-Tol & $p-\mathrm{BrC}_{6} \mathrm{H}_{4}$ & $S$ & $2 \mathbf{i}$ & 55 \\
\hline $1 b$ & $\mathrm{HCF}_{2} \mathrm{CF}_{2}$ & $p$-Tol & $\mathrm{Ph}$ & S & $2 \mathbf{j}$ & 63 \\
\hline $1 b$ & $\mathrm{HCF}_{2} \mathrm{CF}_{2}$ & $p$-Tol & $p-\mathrm{MeOC}_{6} \mathrm{H}_{4}$ & $\mathrm{~S}$ & $2 \mathbf{k}$ & 53 \\
\hline $1 b$ & $\mathrm{HCF}_{2} \mathrm{CF}_{2}$ & $p$-Tol & $p-\mathrm{BrC}_{6} \mathrm{H}_{4}$ & S & 21 & 50 \\
\hline 1c & $\mathrm{CF}_{3}$ & $\mathrm{Et}_{2} \mathrm{~N}$ & $\mathrm{Ph}$ & $\mathrm{O}$ & $2 m$ & 82 \\
\hline 1c & $\mathrm{CF}_{3}$ & $\mathrm{Et}_{2} \mathrm{~N}$ & $p-\mathrm{MeOC}_{6} \mathrm{H}_{4}$ & $\mathrm{O}$ & $2 n$ & 83 \\
\hline 1d & $\mathrm{HCF}_{2} \mathrm{CF}_{2}$ & $\mathrm{Et}_{2} \mathrm{~N}$ & $\mathrm{Ph}$ & $\mathrm{O}$ & 20 & 78 \\
\hline 1d & $\mathrm{HCF}_{2} \mathrm{CF}_{2}$ & $\mathrm{Et}_{2} \mathrm{~N}$ & $p-\mathrm{MeOC}_{6} \mathrm{H}_{4}$ & $\mathrm{O}$ & $2 p$ & 75 \\
\hline 1c & $\mathrm{CF}_{3}$ & $\mathrm{Et}_{2} \mathrm{~N}$ & $\mathrm{Ph}$ & $\mathrm{S}$ & $2 q$ & 61 \\
\hline 1d & $\mathrm{HCF}_{2} \mathrm{CF}_{2}$ & $\mathrm{Et}_{2} \mathrm{~N}$ & $\mathrm{Ph}$ & $\mathrm{S}$ & $2 r$ & 59 \\
\hline
\end{tabular}

${ }^{\mathrm{a}}$ Isolated yield.

Similarly to the Biginelli reaction of fluorinated $\beta$-dicarbonyl compounds 4-hydroxycontaining tetrahydropyrimidin(thi)ones were formed in cyclocondensation of ketones $\mathbf{1}$, aryl aldehydes and urea and no dehydration products of $\mathbf{2}$ have been detected. ${ }^{17-20}$ Initially we used the reaction conditions, which have been proven suitable for the Biginelli reaction of 1,3dicarbonyl compounds involving refluxing reactants in acetonitrile with catalytic amounts of trimethylsilyl chloride as a catalyst. ${ }^{19,21,22}$ However, such procedure appeared to be unappropriate on the reason of poor yields of 2 , which consisted in our case at best 15-25\%. The main cause of the low yields was associated with the tendency of the starting polyfluoroalkyl keto compounds 1 
to form unreactive hydrates with water, evolved during the condensation. Therefore, to enhance the productivity of the cyclocondensation not only anhydrous conditions were required but also elimination of water from the reaction media was essential. We have disclosed that the most favorable Biginelli reaction conditions for the synthesis of $\mathbf{2}$ were heating the starting compounds in a mixture of acetic acid with acetic anhydride as an agent for trapping water which enabled to improve the yields up to $50-85 \%$. Thiourea was also used in place of urea providing corresponding thiones generally in lower yields in comparison with oxo compounds. Tetrahydropyrimidin(thi)ones $\mathbf{2}$ precipitated directly from the reaction mixtures upon heating and the solids were collected by simple filtration and obtained in pure form (>96\% according to LCMS data).

Tetrahydropyrimidin(thi)ones 2 contain three stereogenic centers assuming the possibility to exist as four diastereomers. However for all described compounds according to the NMR data of the crude solid materials only one set of signals was observed, confirming the isolation of the products as single diastereomers. The remarkable feature of the Biginelli reaction to give exclusively one diastereomeric tetrahydropyrimidinone among other possible is described for the condensations of fluoroalkyl $\beta$-diketo compounds. ${ }^{17-20}$ Common peculiarity of the reported Biginelli products, substituted at the 5th position of the tetrahydropyrimidinone cycle with acyl or carbalkoxy group in place of sulfonyl moiety is occurrence of the vicinal coupling between $\mathrm{H}$ 5 and $H-6$ cyclic protons with ${ }^{3} J_{\mathrm{HH}} \sim 11 \mathrm{~Hz}$, allowing unambigously establish trans orientation of the substituents at $C-5$ and $C$ - 6 . As follows from the ${ }^{1} \mathrm{H}$ NMR spectra of compounds 2 no coupling was observed between $H-5$ and $H-6$ protons, which were assigned by the COSY and HETCOR experiments. Signal due to $H-5$ proton exhibited singlet at 4.0-4.2 ppm, whereas $H-6$ proton was coupled with amide proton $\mathrm{NH}-1$ showing doublet at the corresponding region with coupling constant ${ }^{3} \mathrm{~J}_{\mathrm{HH}} 3 \div 4 \mathrm{~Hz}$. Hence the observed coupling constants values for cyclic protons did not allow to determine the orientation of substituents in compounds $\mathbf{2}$ based only on NMR spectral data.

The stereochemistry of tetrahydropyrimidin(thi)ones $\mathbf{2}$ was established on the example of the X-ray diffraction analysis of compound 2a (Fig. 1). Substituents at $C-5$ (tosyl group) and $C-6$ (phenyl group) in the molecule of 2a occupy positions trans to each other similarly to the literary described cases of 5-acyl or 5-carbalkoxy Biginelli tetrahydropyrimidinones. ${ }^{17-20}$ However observed dihedral angle between $H-5$ and $H-6$ protons $(H-C 15-C 16-H$ on Fig. 1$)$ was $84.5^{\circ}$ and in accordance with the Karplus equation ${ }^{23}$ agreed very well with either small or absence of coupling constant for the corresponding protons in ${ }^{1} \mathrm{H}$ NMR spectrum of 2a. In ${ }^{13} \mathrm{C}$ NMR spectra compounds 2a-f,m-p displayed signals due to carbonyl group at $154 \mathrm{ppm}$ while shifts due to

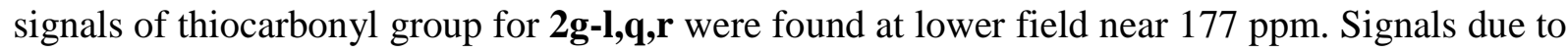
characteristic for tetrahydropirimidin(thi)ones structures quaternary $C-4$ atom of the pyrimidine framework in the ${ }^{13} \mathrm{C}$ NMR spectra occured as quartet $\left({ }^{2} J_{\mathrm{CF}} 27 \div 33 \mathrm{~Hz}\right)$ at $79-82 \mathrm{ppm}$. The results of X-ray analysis obtained for $\mathbf{2 a}$ along with NMR data of the rest synthesized compounds allow to spread the conclusion about the relative stereochemistry around all tetrahydropyrimidin(thi)ones $\mathbf{2}$ (except $\mathbf{2 h}$, see below). 


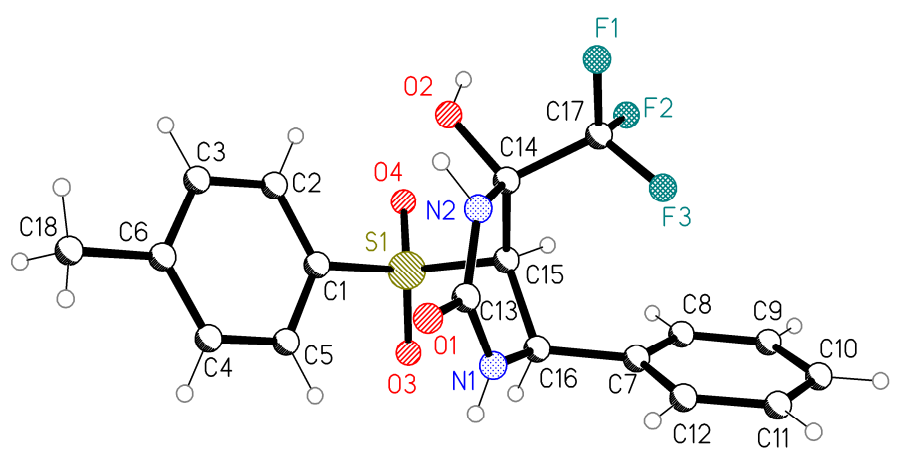

Figure 1. Molecular structure of $\mathbf{2 a}$.

The IR spectra of tetrahydropyrimidinones 2a-f,m-p displayed absorption bands characteristic for $\mathrm{C}=\mathrm{O}\left(1700-1670 \mathrm{~cm}^{-1}\right)$, NH $\left(3450-2970 \mathrm{~cm}^{-1}\right)$ and $\mathrm{SO}_{2}\left(1280-1240 \mathrm{~cm}^{-1}\right)$ functions. Absence of the sharp band at $3400 \mathrm{~cm}^{-1}$ can be explained by the intramolecular hydrogen bonding of $\mathrm{OH}$ proton with carbonyl oxygen of another molecule in solid state which was confirmed by X-ray investigation of 2a. It is noteworthy that tetrahydropyrimidinone $\mathbf{2 a}$ crystallized as a mixture of two crystalline modifications exhibiting different melting points values and distinct types of intermolecular hydrogen bonds in crystals. Their X-Ray analysis data will be reported elsewhere.

Since compounds 2 were isolated in $50-85 \%$ yields as single diastereomer we then examined mother solutions after separation of the precipitates. ${ }^{1} \mathrm{H}$ NMR spectra of the residues obtained after removal of the solvent from the filtrates revealed a set of signals which can assigned to another one diastereomeric Biginelli product for all cases. These minor isomers were not isolated in pure state but their NMR data studies allowed us to conclude about their structures.

The secondary isomeric tetrahydropyrimidin(thi)ones can be easily distinguished from basic ones by the characteristic location of signals due to $H-5$ and $H-6$ cyclic protons in the ${ }^{1} \mathrm{H}$ NMR spectra appearing at 4.4 and $4.7 \mathrm{ppm}$ as doublets with ${ }^{3} J_{\mathrm{HH}} 6 \div 9 \mathrm{~Hz}$ as well as by the differing location of signals for fluoroalkyl groups in ${ }^{19} \mathrm{~F}$ NMR spectra. In particular, for trifluoromethyl-containing compounds 2 in the ${ }^{19} \mathrm{~F}$ NMR spectra chemical shifts due to $\mathrm{CF}_{3}$ group for minor isomers which can be found at around $-76 \mathrm{ppm}$ are contrasting with those occurring for the basic isomers lying at around $-82 \mathrm{ppm}$. The most significant differences of chemical shifts between isomeric tetrahydropyrimidin(thi)ones in the ${ }^{13} \mathrm{C}$ NMR spectra were observed for the resonances of $C-6$ cyclic carbon and the neighbored $C$-ipso aromatic carbon which were downshifted by 3-5 ppm compared to the signals of major products. The obtaining of the single isomer in precipitates of $\mathbf{2}$ can be explained by its predominating solubility comparing with minor isomer. Having synthesized a series of Biginelli products $\mathbf{2}$ with various combinations of substituents in the ring we have revealed that in case of compound $\mathbf{2 h}$ the product precipitated from the reaction mixture had NMR spectral characteristics typical for those of minor isomers of the rest tetrahydropyrimidin(thi)ones. 
Another one-pot method described here for the preparation of polyfluoroalkyl pyrimidinones bearing sulfur moieties involves three-component reactions of 2-oxo-2polyfluoroalkylethane-1-sulfones and -sulfamides $\mathbf{1}$ with urea and trialkyl orthoformate. Previously $^{24}$ we have shown that $\beta$-polyfluoroalkyl- $\beta$-ketophosphonates undergo threecomponent cyclocondensation reaction with trialkyl orthoformate and urea giving phosphonylcontaining 3,4-dihydropyrimidin-2(1H)-ones. The advantage of the elaborated one-pot procedure was its simplicity, avoiding isolation of intermediates and use of additional reagents to accomplish cyclization step to heterocyclic core. Having these results, another one-pot procedure was proposed which opens straightforward access to the sulfonyl-containing pyrimidinones by the similar three-component cyclocondensation reaction of ketones 1. It was found that heating 2-oxo-2-polyfluoroalkylethane-1-sulfones 1a,b and -sulfamide 1e with urea and excess trialkyl orthoformate during 3 hours led to the formation of sulfonyl-substituted 3,4-dihydropyrimidin2(1H)-ones 3a-c (Scheme 1). Compounds 3 solidified from the reaction mixture under heating and were obtained virtually pure (>95\% according to LC-MS data) in $65-85 \%$ overall yields. It is noteworthy that on the contrary with the Biginelli reaction of ketosulfones and ketosulfamides three-component reaction with urea and trialkyl orthoformate proceeded even with hydrtated ketones.

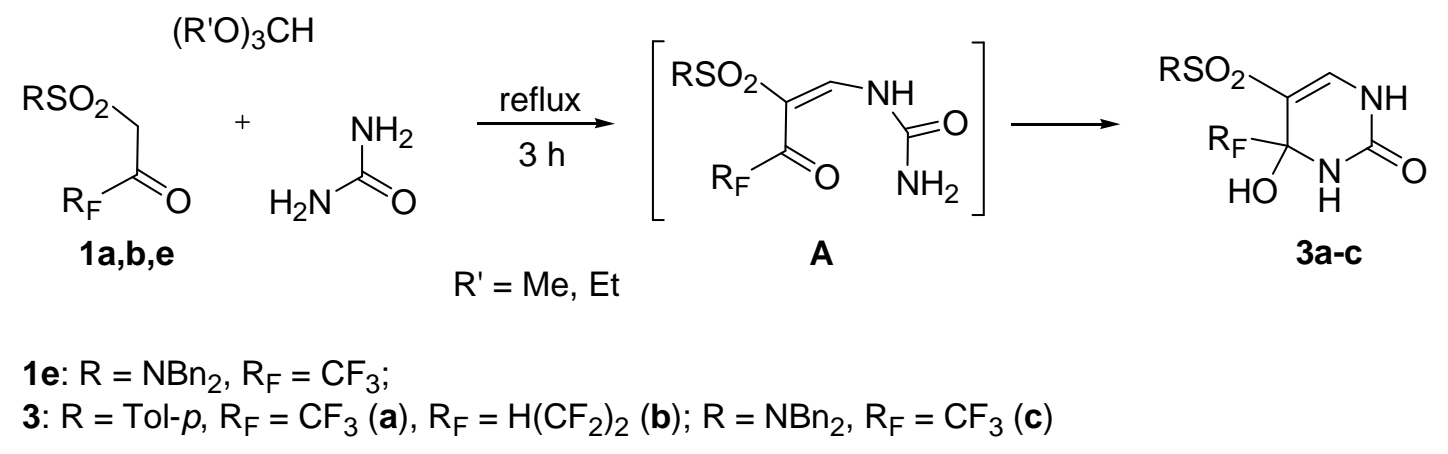

Scheme 1. Three-component reaction of 2-oxo-2-polyfluoroalkylethane-1-sulfones $\mathbf{1 a , b}$ and sulfamide 1e with trialkyl orthoformate and urea.

In the course of this sequential one-pot protocol $\alpha$-alkoxyvinyl ketones formed via condensation of $\mathbf{1}$ with trialkyl orthoformate reacted with urea to give acyclic intermediate $\mathbf{A}$, which underwent instantaneous intramolecular cyclization resulting in compounds 3 (Scheme 1). For similar reactions of $\beta$-dicarbonyl compounds reported in the literature the construction of a pyrimidine ring required two consequtive stages as well as the isolation of acyclic precursors, cyclization to pyrimidinones on the final step being achieved under rigorous conditions and the use of base. ${ }^{25-26}$

In order to broaden the scope of the developed three-component reaction we have shown that treatment of 2-oxo-2-polyfluoroalkylethane-1-sulfones 1a,b and -sulfamide 1c with excess trialkyl orthoformate and heterocyclic amine (instead of urea) such as 2-aminobenzimidazole 
afforded upon heating 4,10-dihydropyrimido[1,2-a]benzimidazoles 4 in 45-55\% yields (Scheme 2) similarly to the one-pot reactions described for non-fluorinated $\beta$-ketosulfones leading to the fused heterocyclic compounds, which were tested for Aurora-A kinase inhibitors. ${ }^{27}$ Structures of 4a-c were supported by the NMR spectral data and mass-spectrometry data of the isolated compounds.

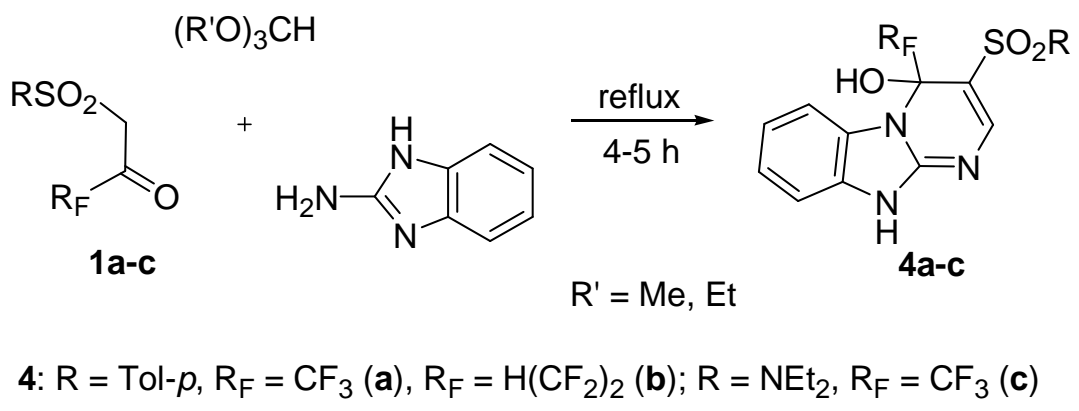

Scheme 2. Three-component reaction of 2-oxo-2-polyfluoroalkylethane-1-sulfones 1a,b and sulfamide 1c with trialkyl orthoformate and 2-aminobenzimidazole.

Next our investigations were directed to the exploration of some chemical properties of the obtained heterocycles with the aim of the expanding their synthetic potential.

Dihydropyrimidinone 3a was readily dehydrated upon heating with excess phosphorus pentoxide in acetonitrile to form non-isolable pyrimidine derivative $\mathbf{5}$, which due to the highly electrophilic $\mathrm{C}=\mathrm{N}$ bond ${ }^{28}$ spontaneously reacted with traces of water furnishing starting pyrimidinone 3a. Relative readiness to eliminate water for 3a in comparison with the remarkable stability towards dehydration of Biginelli compounds 2 , which we eventually failed to dehydrate with various dehydrating agents, could account for the appearance of aromatic heterocyclic ring in 5. Trapping intermediate $\mathbf{5}$ with nucleophiles such as alcohols afforded 4-alkoxy-3,4dihydropyrimidin-2(1H)-ones 6 (Scheme 3). Reactions were easily controlled by the ${ }^{19} \mathrm{~F}$ NMR spectroscopy, in particular after refluxing 3a with excess of $\mathrm{P}_{2} \mathrm{O}_{5}$ in the ${ }^{19} \mathrm{~F}$ NMR spectrum of the reaction mixture the resonance peak due to trifluoromethyl group at $-81 \mathrm{ppm}$ of $\mathbf{3 a}$ disappeared to give another peak at $-66 \mathrm{ppm}$ corresponding to the structure of the dehydrated derivative $\mathbf{5}$. The latter intermediate without isolation was reacted with several alcohols to give appropriate 4alkoxy dihydropyrimidinones $\mathbf{6 a - c}$, displaying signals due to $\mathrm{CF}_{3}$ group at $-78 \div-79 \mathrm{ppm}$ in the ${ }^{19}$ F NMR spectra. 


$$
\text { 6: } \mathrm{R}=\mathrm{Me}(\mathbf{a}) ; \mathrm{EtO}_{m,(\mathbf{m}) ;}^{\mathrm{Me}}
$$

Scheme 3. Dehydratation-alcohol addition reactions for compound 3a.

Since addition of nucleophiles to the intermediate pyrimidinone 5 resulted in the appearance of stereogenic center, use of alcohols containing asymmetric carbon atom could generate diastereomers. However, upon addition of asymmetric alcohols such as optically active ethyl $L$-(-)-lactate or racemic (DL)-sec-phenethyl alcohol to $\mathbf{5}$ formation of only one diastereomer was observed for both cases $\mathbf{6 b}, \mathbf{c}$ as deduced from ${ }^{19} \mathrm{~F}$ NMR spectral data of the reaction mixtures showing single peaks due to $\mathrm{CF}_{3}$ group. This fact may be regarded as evidence of the asymmetric induction in reactions of $\mathbf{5}$ with asymmetric alcohols.

Treatment of dihydropyrimidinone 3a with a two-fold excess of potassium hydroxide in methanol resulted in the dehydration product and the formation of potassium salt 7 which was isolated and identified by the NMR spectral data. Our efforts to alkylate 7 with alkyl halides, dimethylsulfate or acylate it with acyl halides were unsuccessful. Compound $\mathbf{7}$ appeared hygroscopic and easily underwent hydrolysis and addition of water, therefore after acidification of 7 with conc. hydrochloric acid starting dihydropyrimidinone 3a was recovered in almost quantitative yield (Scheme 4).

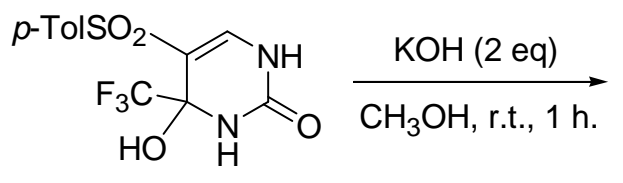

3a

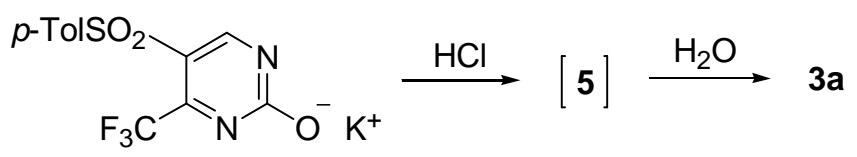

7

Scheme 4. Reaction of compound 3a with base.

In contrast to the previous case, reaction of Biginelli tetrahydropyrimidinone 2a with inorganic $(\mathrm{KOH}, \mathrm{NaH})$ as well as organic $\left(\mathrm{Et}_{3} \mathrm{~N}, t-\mathrm{BuOK}, \mathrm{MeONa}\right)$ bases resulted in the cleavage of the pyrimidinone cycle on $C-4-C-5$ bond to yield acyclic derivative 8 regardless of the base applied. ${ }^{19} \mathrm{~F}$ NMR spectrum of the reaction mixture in methanol taken after reacting $\mathbf{2 a}$ with potassium hydroxide revealed a signal at $-75 \mathrm{ppm}$ assignable to trifluoromethyl group attached to $s p^{2}$-carbon atom. However after aqueous work-up of the reaction mixture a compound was isolated in $70 \%$ yield which did not contain fluorine substituent. Based on NMR specral data the product was assigned to 1-(1-phenyl-2-tosylethyl)urea $\mathbf{8}$, formed evidently after 
loss of potassium trifluoroacetate which was detected in ${ }^{19} \mathrm{~F}$ NMR spectrum of the reaction mixture (Scheme 5).<smiles>O=C1NC(c2ccccc2)[C@H](O[Se]O)C(O)(C(F)(F)F)N1</smiles>

$2 a$

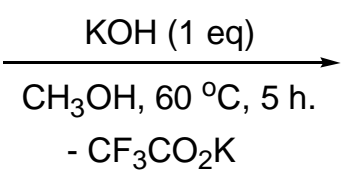

$-\mathrm{CF}_{3} \mathrm{CO}_{2} \mathrm{~K}$<smiles>NC(=O)NC(COS(=O)(=O)O)c1ccccc1</smiles>

8

Scheme 5. Reaction of compound 2a with base leading to the opening of cycle.

We have tried also to modify pyrimidinone derivatives $\mathbf{2 a}$ and $\mathbf{3 a}$ by the conversion of heterocyclic amide moiety into imidoyl chloride fragment using reactions with phosphorus pentachloride.

Dihydropyrimidinone 3a was refluxed with equimolar amount of phosphorus pentachloride in acetonitrile with monitoring the reaction progress by ${ }^{31} \mathrm{P}$ NMR spectroscopy, indicating complete consumption of $\mathrm{PCl}_{5}$ in the course of reaction. In ${ }^{19} \mathrm{~F}$ NMR spectrum of the reaction mixture signal at $-82 \mathrm{ppm}$ due to trifluoromethyl group of 3a disappeared while a new peak arose at $-65 \mathrm{ppm}$. These observations can indicate the formation of chloropyrimidine derivative 9, however we failed in attempts neither to isolate it for characterization nor trap with nucleophiles since it was extremely hygroscopic and easily converted to starting pyrimidinone $\mathbf{3 a}$ (Scheme 6).

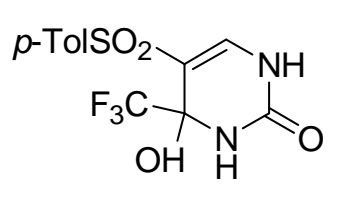

3a

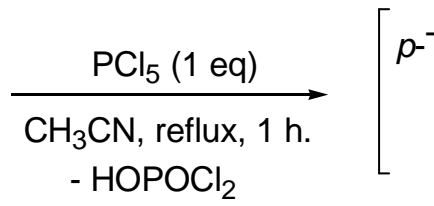

- $\mathrm{HOPOCl}_{2}$

Scheme 6. Reaction of compound 3a with $\mathrm{PCl}_{5}$.

Reaction of equimolar amounts of tetrahydropyrimidinone 2a with phosphorus pentachloride gave unseparable mixture of products. At the same time after refluxing suspension of compound 2a with two-fold excess of $\mathrm{PCl}_{5}$ in benzene 1,1,1-trifluoro-4-phenyl-3-tosylbut-3en-2-one $\mathbf{1 0}$ was isolated in $75 \%$ yield from the reaction mixture, formed as a result of pyrimidinone cycle cleavage. Structure of compound $\mathbf{1 0}$ was supported unequivocally by the NMR data, mass-spectrometry and elemental analysis (Scheme 7). It should be noted that benzylidene derivative $\mathbf{1 0}$ is formally a product of the Knoevenagel condensation of 1,1,1trifluoro-3-tosylpropan-2-one 1a with benzaldehyde which has been recently obtained. ${ }^{29}$ 


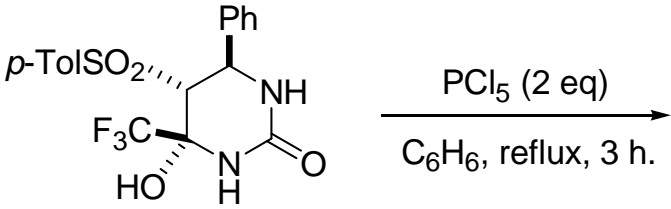

$2 a$<smiles>O=C(C(=Cc1ccccc1)OS(=O)(=O)[O-])C(F)(F)F</smiles>

10

Scheme 7. Reaction of compound $2 \mathbf{a}$ with $\mathrm{PCl}_{5}$.

\section{Conclusions}

We have elaborated convenient one-pot three-component reactions of 2-oxo-2polyfluoroalkylethane-1-sulfones and -sulfamides leading to a series of pyrimidine derivatives. Optimal conditions for the Biginelli reaction of the studied ketones have been found involving use of acetic acid-acetic anhydride mixture not only as solvent but also as dehydrating agent for the removal of water evolved during the condensation. Additionally, certain chemical properties of the obtained heterocyclic compounds have been examined.

\section{Experimental Section}

General. Melting points were measured on a Boetius melting point devise and are uncorrected. IR spectra were recorded on a UR-20 spectrometer with samples in $\mathrm{KBr}$ disks. ${ }^{19} \mathrm{~F}$ and ${ }^{31} \mathrm{P}$ NMR spectra were recorded at 188.14 and $80.95 \mathrm{MHz}$ respectively on a Varian Gemini-200 spectrometer with $\mathrm{C}_{6} \mathrm{~F}_{6}\left(\delta_{\mathrm{F}}=-162.9 \mathrm{ppm}\right.$ relative to $\left.\mathrm{CFCl}_{3}\right)$ and $\mathrm{H}_{3} \mathrm{PO}_{4}\left(\delta_{\mathrm{P}}=0.0 \mathrm{ppm}\right)$ as standards. ${ }^{1} \mathrm{H}$ NMR spectra were recorded at 299.94 or $400.13 \mathrm{MHz}$ on a Varian VXR-300 or Bruker Avance 400 spectrometers respectively, ${ }^{13} \mathrm{C}$ NMR, APT, ${ }^{1} \mathrm{H}-{ }^{1} \mathrm{H}$ COSY,${ }^{13} \mathrm{C}-{ }^{1} \mathrm{H}$ HETCOR spectra were recorded at $100.62 \mathrm{MHz}$ for ${ }^{13} \mathrm{C}$ and at $400.13 \mathrm{MHz}$ for ${ }^{1} \mathrm{H}$ on a Bruker Avance 400 spectrometer. NMR spectra (except ${ }^{31} \mathrm{P}$ ) were obtained in DMSO- $d_{6}$ or acetone- $d_{6}$ solutions with the solvent residual peak as reference $\left(\mathrm{DMSO}-d_{6} \delta_{\mathrm{H}}=2.50 \mathrm{ppm}, \delta_{\mathrm{C}}=39.52 \mathrm{ppm}\right.$ and acetone- $d_{6}$ $\left.\delta_{\mathrm{H}}=2.05 \mathrm{ppm}, \delta_{\mathrm{C}}=29.84 \mathrm{ppm}\right)$. HPLC-MS analyses were carried out using a system consisting of an Agilent 1100 Series high performance liquid chromatograph equipped with a diode-matrix and an Agilent LC/MSD SL mass selective detector (ionization method: chemical ionization under atmospheric pressure (APCI)). MS data were also obtained on the Hewlett-Packard 589015972 apparatus (GC/MS) operating at an ionization potential of $70 \mathrm{eV}$ in the electron impact (EI) mode.

General synthetic procedure for the Biginelli reaction of 2-oxo-2-polyfluoroalkylethane-1sulfones (1a,b) and -sulfamides (1c,d). Compounds 2a-r. To a mixture of urea (66 mg, 1.1 $\mathrm{mmol}$ ) or thiourea ( $84 \mathrm{mg}, 1.1 \mathrm{mmol})$ and the corresponding aryl aldehyde $(1.1 \mathrm{mmol})$ a solution 
of ketosulfone 1a,b $(1 \mathrm{mmol})$ or ketosulfamide 1c,d $(1 \mathrm{mmol})$ in a mixture of $\mathrm{Ac}_{2} \mathrm{O}(1.5 \mathrm{~mL}, 1.5$ mmol) and glacial $\mathrm{AcOH}(1 \mathrm{~mL})$ was added and the resulting mixture was heated at $80{ }^{\circ} \mathrm{C}$ for 5 $\mathrm{h}$. White solid precepitated from the reaction mixture within this period, then the suspension was allowed to cool, diluted with $\mathrm{Et}_{2} \mathrm{O}(2 \mathrm{~mL})$, the precipitate filtered, washed with $\mathrm{H}_{2} \mathrm{O}, \mathrm{Et}_{2} \mathrm{O}$ and dried to give tetrahydropyrimidinones $\mathbf{2 a - r}$ as colorless or white solids which were crystallized from an appropriate solvent.

\section{4-Hydroxy-6-phenyl-5-[(4-methylphenyl)sulfonyl]-4-(trifluoromethyl)-tetrahydropyrimi-} din-2(1H)-one (2a). Colorless cubes, yield 85\%, $352 \mathrm{mg}, \mathrm{mp} 225{ }^{\circ} \mathrm{C}$ (from $\mathrm{CH}_{3} \mathrm{CN}$ ). Spectral and analytical data of compound $\mathbf{2 a}$ have been reported previously.

\section{4-Hydroxy-6-(4-methoxyphenyl)-5-[(4-methylphenyl)sulfonyl]-4-(trifluoromethyl)-tetra-} hydropyrimidin-2(1H)-one (2b). Colorless needles, yield 83\%, $368 \mathrm{mg}, \mathrm{mp} 194-195{ }^{\circ} \mathrm{C}$ (from EtOH); IR $\left(v_{\max }, \mathrm{cm}^{-1}\right): 3400,3330,3250,3110(\mathrm{NH}, \mathrm{OH}), 1690(\mathrm{C}=\mathrm{O}) .{ }^{1} \mathrm{H}$ NMR $(300 \mathrm{MHz}$, DMSO- $\left.d_{6}\right): \delta_{\mathrm{H}} 7.38$ and $7.81\left(4 \mathrm{H}, \mathrm{dd}, J 8.4 \mathrm{~Hz}, \mathrm{C}_{6} \mathrm{H}_{4}-\right.$ para $), 7.63(1 \mathrm{H}, \mathrm{s}, \mathrm{OH}), 7.57(1 \mathrm{H}, \mathrm{d}, J 3.3$ $\mathrm{Hz}, \mathrm{NH}-1), 6.86$ and $7.14\left(4 \mathrm{H}, \mathrm{dd}, J 8.1 \mathrm{~Hz}, \mathrm{C}_{6} \mathrm{H}_{4}\right.$-para $), 6.93(1 \mathrm{H}, \mathrm{s}, \mathrm{NH}-3), 5.37(1 \mathrm{H}, \mathrm{d}, J 3.3$ $\mathrm{Hz}, \mathrm{H}-6), 4.12(1 \mathrm{H}, \mathrm{s}, \mathrm{H}-5), 3.73\left(3 \mathrm{H}, \mathrm{s}, \mathrm{OCH}_{3}\right), 2.40\left(3 \mathrm{H}, \mathrm{s}, \mathrm{CH}_{3}\right) .{ }^{13} \mathrm{C} \mathrm{NMR}(100 \mathrm{MHz}$, DMSO- $\left.d_{6}\right): \delta_{\mathrm{C}} 158.4\left(\underline{\mathrm{C}}_{\mathrm{Ar}}-\mathrm{OCH}_{3}\right), 153.7(\mathrm{C}=\mathrm{O}), 143.8\left(\underline{\mathrm{C}}_{\mathrm{Ar}}-\mathrm{CH}_{3}\right), 138.0\left(\mathrm{C}_{\mathrm{Ar}}-\mathrm{SO}_{2}\right), 132.0\left(\mathrm{C}_{\mathrm{Ar}}\right)$, 129.1, 128.6, $126.9\left(3 \mathrm{C}_{\mathrm{Ar}}-\mathrm{H}\right), 122.4\left(\mathrm{q}, J_{\mathrm{CF}} 288.0 \mathrm{~Hz}, \mathrm{CF}_{3}\right), 113.6\left(\mathrm{C}_{\mathrm{Ar}}\right), 81.0\left(\mathrm{q},{ }^{2} J_{\mathrm{CF}} 33.0 \mathrm{~Hz}, \mathrm{C}-\right.$ 4), $66.3(\mathrm{C}-5), 55.0\left(\mathrm{OCH}_{3}\right), 49.7(\mathrm{C}-6), 20.9\left(\mathrm{CH}_{3}\right) .{ }^{19} \mathrm{~F}$ NMR $\left(188 \mathrm{MHz}, \mathrm{DMSO}-d_{6}\right): \delta_{\mathrm{F}}-82.58$ $\left(3 \mathrm{~F}, \mathrm{~s}, \mathrm{CF}_{3}\right)$. MS APCI, $m / z(\%)=445(\mathrm{MH}, 100)$. Anal. Calcd for $\mathrm{C}_{19} \mathrm{H}_{19} \mathrm{~F}_{3} \mathrm{~N}_{2} \mathrm{O}_{5} \mathrm{~S}$ (444.42): $\mathrm{C}$, $51.35 ; \mathrm{H}, 4.31 ; \mathrm{N}, 6.30 ; \mathrm{S}, 7.21 \%$. Found: C, 51.24; H, 4.40; N, 6.25; S, 7.29\%.

6-(4-Bromophenyl)-4-hydroxy-5-[(4-methylphenyl)sulfonyl]-4-(trifluoromethyl)tetrahydropyrimidin-2(1H)-one (2c). Colorless solid, yield 77\%, $380 \mathrm{mg}, \mathrm{mp} 215-217^{\circ} \mathrm{C}$ (from $\mathrm{CH}_{3} \mathrm{CN}$ ); IR $\left(v_{\max }, \mathrm{cm}^{-1}\right): 3390,3320,3240,3110(\mathrm{NH}, \mathrm{OH}), 1695(\mathrm{C}=\mathrm{O}) .{ }^{1} \mathrm{H}$ NMR (300 MHz, DMSO$\left.d_{6}\right): \delta_{\mathrm{H}} 7.38$ and $7.83\left(4 \mathrm{H}, \mathrm{dd}, J 8.4 \mathrm{~Hz}, \mathrm{C}_{6} \mathrm{H}_{4}\right.$-para $), 7.67(1 \mathrm{H}, \mathrm{s}, \mathrm{OH}), 7.66(1 \mathrm{H}, \mathrm{d}, J 3.7 \mathrm{~Hz}$, NH-1), 7.05 and $7.51\left(4 \mathrm{H}, \mathrm{dd}, J 8.1 \mathrm{~Hz}, \mathrm{C}_{6} \mathrm{H}_{4}\right.$-para $), 7.00(1 \mathrm{H}, \mathrm{s}, \mathrm{NH}-3), 5.41(1 \mathrm{H}, \mathrm{d}, J 3.7 \mathrm{~Hz}$, $\mathrm{H}-6), 4.20(1 \mathrm{H}, \mathrm{s}, \mathrm{H}-5), 2.41\left(3 \mathrm{H}, \mathrm{s}, \mathrm{CH}_{3}\right) .{ }^{13} \mathrm{C}$ NMR (100 MHz, DMSO- $\left.d_{6}\right): \delta_{\mathrm{C}} 153.5(\mathrm{C}=\mathrm{O})$, $143.9\left(\underline{\mathrm{C}}_{\mathrm{Ar}}-\mathrm{CH}_{3}\right), 139.6\left(\mathrm{C}_{\mathrm{Ar}}\right), 137.9\left(\mathrm{C}_{\mathrm{Ar}}-\mathrm{SO}_{2}\right), 131.0,129.1,128.6,128.0\left(4 \mathrm{C}_{\mathrm{Ar}}-\mathrm{H}\right), 122.3(\mathrm{q}$, $\left.J_{\mathrm{CF}} 288.3 \mathrm{~Hz}, \mathrm{CF}_{3}\right), 120.4\left(\mathrm{C}_{\mathrm{Ar}}-\mathrm{Br}\right), 80.9\left(\mathrm{q},{ }^{2} J_{\mathrm{CF}} 32.3 \mathrm{~Hz}, \mathrm{C}-4\right), 65.7$ (C-5), 49.7 (C-6), 20.9 $\left(\mathrm{CH}_{3}\right) .{ }^{19} \mathrm{~F}$ NMR $\left(188 \mathrm{MHz}, \mathrm{DMSO}-d_{6}\right): \delta_{\mathrm{F}}-82.52\left(3 \mathrm{~F}, \mathrm{~s}, \mathrm{CF}_{3}\right)$. MS APCI, $m / z(\%)=494$ and 492 (MH, 100 and 95). Anal. Calcd for $\mathrm{C}_{18} \mathrm{H}_{16} \mathrm{BrF}_{3} \mathrm{~N}_{2} \mathrm{O}_{4} \mathrm{~S}$ (493.29): C, 43.83; H, 3.27; N, 5.68; S, 6.50\%. Found C, 43.93; H, 3.36; N, 5.77; S, 6.64\%.

4-Hydroxy-5-[(4-methylphenyl)sulfonyl]-6-phenyl-4-(1,1,2,2-tetrafluoroethyl)-tetrahydropyrimidin-2(1H)-one (2d). Colorless solid, yield 80\%, $357 \mathrm{mg}, \mathrm{mp} 162-164{ }^{\circ} \mathrm{C}$ (from $\mathrm{CH}_{3} \mathrm{CN}$ ); IR $\left(v_{\max }, \mathrm{cm}^{-1}\right)$ : 3450, 3220, 3080, $2980(\mathrm{NH}, \mathrm{OH}), 1670(\mathrm{C}=\mathrm{O}) .{ }^{1} \mathrm{H}$ NMR (300 MHz, DMSO$\left.d_{6}\right): \delta_{\mathrm{H}} 7.41$ and $7.85\left(4 \mathrm{H}, \mathrm{dd}, J 7.8 \mathrm{~Hz}, \mathrm{C}_{6} \mathrm{H}_{4}\right.$-para $), 7.61(2 \mathrm{H}, \mathrm{m}, \mathrm{NH}-1+\mathrm{OH}), 7.18-7.34(5 \mathrm{H}$, $\left.\mathrm{m}, \mathrm{C}_{6} \mathrm{H}_{5}\right), 6.51(1 \mathrm{H}, \mathrm{s}, \mathrm{NH}-3), 6.29\left(1 \mathrm{H}, \mathrm{tt},{ }^{2} J_{\mathrm{HF}} 52.0 \mathrm{~Hz},{ }^{3} J_{\mathrm{HF}} 5.4 \mathrm{~Hz}, \mathrm{HCF}_{2}\right), 5.44(1 \mathrm{H}, \mathrm{d}, J 3.3$ $\mathrm{Hz}, \mathrm{H}-6), 4.23(1 \mathrm{H}, \mathrm{s}, \mathrm{H}-5), 2.41\left(3 \mathrm{H}, \mathrm{s}, \mathrm{CH}_{3}\right) .{ }^{13} \mathrm{C}$ NMR (100 MHz, DMSO- $\left.d_{6}\right): \delta_{\mathrm{C}} 153.7$ $(\mathrm{C}=\mathrm{O}), 144.1\left(\underline{\mathrm{C}}_{\mathrm{Ar}}-\mathrm{CH}_{3}\right), 140.5\left(\mathrm{C}_{\mathrm{Ph}}-\right.$ ipso $), 137.7\left(\mathrm{C}_{\mathrm{Ar}^{-}}-\mathrm{SO}_{2}\right), 129.0,128.7,128.1,127.0,125.5$ $\left(5 \mathrm{C}_{\mathrm{Ar}}-\mathrm{H}\right), 111-118\left(\mathrm{~m}, \mathrm{CF}_{2}\right), 108.6\left(\mathrm{tt}, J_{\mathrm{CF}} 247.0 \mathrm{~Hz},{ }^{2} J_{\mathrm{CF}} 29.2 \mathrm{~Hz}, \mathrm{HCF}_{2}\right), 82.1\left(\mathrm{t},{ }^{2} J_{\mathrm{CF}} 27.0 \mathrm{~Hz}\right.$, C-4), $66.1(\mathrm{C}-5), 50.1(\mathrm{C}-6), 21.0\left(\mathrm{CH}_{3}\right) .{ }^{19} \mathrm{~F}$ NMR $\left(188 \mathrm{MHz}, \mathrm{DMSO}-d_{6}\right): \delta_{\mathrm{F}}-127.28(2 \mathrm{~F}, \mathrm{~m}$, 
$\left.\mathrm{CF}_{2}\right), \delta_{\mathrm{A}}-134.48\left(1 \mathrm{~F}, \mathrm{ABd}, J_{\mathrm{AB}} 299.0 \mathrm{~Hz},{ }^{2} J_{\mathrm{FH}} 52.0 \mathrm{~Hz}, \mathrm{HCF}_{\mathrm{A}} \mathrm{F}_{\mathrm{B}}\right), \delta_{\mathrm{B}}-136.02\left(1 \mathrm{~F}, \mathrm{ABd}, J_{\mathrm{AB}}\right.$ $\left.299.0 \mathrm{~Hz},{ }^{2} J_{\mathrm{FH}} 52.0 \mathrm{~Hz}, \mathrm{HCF}_{\mathrm{A}} \underline{\mathrm{F}}_{\mathrm{B}}\right)$. MS APCI, $m / z(\%)=447(\mathrm{MH}, 100)$. Anal. Calcd for $\mathrm{C}_{19} \mathrm{H}_{18} \mathrm{~F}_{4} \mathrm{~N}_{2} \mathrm{O}_{4} \mathrm{~S}$ (446.42): C, 51.12; H, 4.06; N, 6.28; S, 7.18\%. Found: C, 52.10; H, 4.10; N, $5.50 ; \mathrm{S}, 7.25 \%$.

4-Hydroxy-6-(4-methoxyphenyl)-5-[(4-methylphenyl)sulfonyl]-4-(1,1,2,2-tetrafluoroethyl)tetrahydropyrimidin-2(1H)-one (2e). Colorless solid, yield $75 \%$, $357 \mathrm{mg}, \mathrm{mp} 187-189{ }^{\circ} \mathrm{C}$ (from EtOH); IR ( $\left.v_{\max }, \mathrm{cm}^{-1}\right)$ : 3440, 3220, 3080, $2970(\mathrm{NH}, \mathrm{OH}), 1670(\mathrm{C}=\mathrm{O}) .{ }^{1} \mathrm{H}$ NMR $(300$ MHz, DMSO- $\left.d_{6}\right): \delta_{\mathrm{H}} 7.38$ and $7.78\left(4 \mathrm{H}, \mathrm{dd}, J 7.8 \mathrm{~Hz}, \mathrm{C}_{6} \mathrm{H}_{4}-\right.$ para $), 7.62(1 \mathrm{H}, \mathrm{s}, \mathrm{OH}), 7.55(1 \mathrm{H}$, m, NH-1), 6.84 and 7.07 (4H, dd, $J 8.4 \mathrm{~Hz}, \mathrm{C}_{6} \mathrm{H}_{4}-$ para $), 6.53(1 \mathrm{H}, \mathrm{s}, \mathrm{NH}-3), 6.35\left(1 \mathrm{H}, \mathrm{tt},{ }^{2} J_{\mathrm{HF}}\right.$ $\left.51.5 \mathrm{~Hz},{ }^{3} J_{\mathrm{HF}} 5.9 \mathrm{~Hz}, \mathrm{HCF}_{2}\right), 5.33(1 \mathrm{H}, \mathrm{m}, \mathrm{H}-6), 4.18(1 \mathrm{H}, \mathrm{s}, \mathrm{H}-5), 3.72\left(3 \mathrm{H}, \mathrm{s}, \mathrm{OCH}_{3}\right), 2.40(3 \mathrm{H}$, s, $\left.\mathrm{CH}_{3}\right) .{ }^{13} \mathrm{C}$ NMR $\left(100 \mathrm{MHz}, \mathrm{DMSO}-d_{6}\right): \delta_{\mathrm{C}} 158.4\left(\underline{\mathrm{C}}_{\mathrm{Ar}}-\mathrm{OCH}_{3}\right), 153.9(\mathrm{C}=\mathrm{O}), 144.0\left(\underline{\mathrm{C}}_{\mathrm{Ar}}-\mathrm{CH}_{3}\right)$, $137.9\left(\mathrm{C}_{\mathrm{Ar}}-\mathrm{SO}_{2}\right), 132.0\left(\mathrm{C}_{\mathrm{Ar}}\right), 129.3,128.6,127.0\left(3 \mathrm{C}_{\mathrm{Ar}}-\mathrm{H}\right), 113.6\left(\mathrm{C}_{\mathrm{Ar}}\right), 111-118\left(\mathrm{~m}, \mathrm{CF}_{2}\right), 108.8$ (tt, $\left.J_{\mathrm{CF}} 249.0 \mathrm{~Hz},{ }^{2} J_{\mathrm{CF}} 29.0 \mathrm{~Hz}, \mathrm{HCF}_{2}\right), 82.2\left(\mathrm{t},{ }^{2} J_{\mathrm{CF}} 26.9 \mathrm{~Hz}, \mathrm{C}-4\right), 66.3(\mathrm{C}-5), 55.1\left(\mathrm{OCH}_{3}\right), 49.9$ (C-6), $21.0\left(\mathrm{CH}_{3}\right) .{ }^{19} \mathrm{~F}$ NMR (188 MHz, DMSO- $\left.d_{6}\right): \delta_{\mathrm{F}} \delta_{\mathrm{A}}-126.50\left(1 \mathrm{~F}, \mathrm{ABm}, J_{\mathrm{AB}} 264.3 \mathrm{~Hz}\right.$, $\left.\mathrm{CF}_{\mathrm{A}} \mathrm{F}_{\mathrm{B}}\right), \delta_{\mathrm{B}}-127.18\left(1 \mathrm{~F}, \mathrm{ABm}, J_{\mathrm{AB}} 264.3 \mathrm{~Hz}, \mathrm{CF}_{\mathrm{A}} \underline{F}_{\mathrm{B}}\right), \delta_{\mathrm{A}}-134.52\left(1 \mathrm{~F}, \mathrm{ABd}, J_{\mathrm{AB}} 299.9 \mathrm{~Hz},{ }^{2} J_{\mathrm{FH}}\right.$ $\left.51.5 \mathrm{~Hz}, \mathrm{HCF}_{\mathrm{A}} \mathrm{F}_{\mathrm{B}}\right), \delta_{\mathrm{B}}-135.76\left(1 \mathrm{~F}, \mathrm{ABd}, J_{\mathrm{AB}} 299.9 \mathrm{~Hz},{ }^{2} J_{\mathrm{FH}} 51.5 \mathrm{~Hz}, \mathrm{HCF}_{\mathrm{A}} \underline{\mathrm{F}}_{\mathrm{B}}\right) . \mathrm{MS}$ APCI, $m / z$ $(\%)=477(\mathrm{MH}, 100)$. Anal. Calcd for $\mathrm{C}_{20} \mathrm{H}_{20} \mathrm{~F}_{4} \mathrm{~N}_{2} \mathrm{O}_{5} \mathrm{~S}$ (476.44): C, 50.42; H, 4.23; N, 5.88; S, 6.73\%. Found:C, 50.36; H, 4.29; N, 5.97; S, 6.80\%.

6-(4-Bromophenyl)-4-hydroxy-5-[(4-methylphenyl)sulfonyl]-4-(1,1,2,2-tetrafluoroethyl)tetrahydropyrimidin-2(1H)-one (2f). Colorless solid, yield 66\%, $346 \mathrm{mg}, \mathrm{mp}$ 194-196 ${ }^{\circ} \mathrm{C}$ (from $\left.\mathrm{CH}_{3} \mathrm{CN}\right)$; IR $\left(v_{\max }, \mathrm{cm}^{-1}\right): 3395,3325,3240,3100(\mathrm{NH}, \mathrm{OH}), 1695(\mathrm{C}=\mathrm{O}) .{ }^{1} \mathrm{H}$ NMR $(300 \mathrm{MHz}$, DMSO- $\left.d_{6}\right): \delta_{\mathrm{H}} 7.38$ and $7.85\left(4 \mathrm{H}, \mathrm{dd}, J 8.1 \mathrm{~Hz}, \mathrm{C}_{6} \mathrm{H}_{4}-\right.$ para $), 7.67(1 \mathrm{H}, \mathrm{s}, \mathrm{OH}), 7.65(1 \mathrm{H}, \mathrm{d}, J 3.1$ $\mathrm{Hz}, \mathrm{NH}-1), 7.11$ and $7.50\left(4 \mathrm{H}, \mathrm{dd}, J 8.4 \mathrm{~Hz}, \mathrm{C}_{6} \mathrm{H}_{4}-\right.$ para $), 6.63(1 \mathrm{H}, \mathrm{s}, \mathrm{NH}-3), 6.32\left(1 \mathrm{H}, \mathrm{tt},{ }^{2} J_{\mathrm{HF}}\right.$ $\left.52.0 \mathrm{~Hz},{ }^{3} J_{\mathrm{HF}} 6.2 \mathrm{~Hz}, \mathrm{HCF}_{2}\right), 5.41(1 \mathrm{H}, \mathrm{d}, J 3.1 \mathrm{~Hz}, \mathrm{H}-6), 4.20(1 \mathrm{H}, \mathrm{s}, \mathrm{H}-5), 2.41\left(3 \mathrm{H}, \mathrm{s}, \mathrm{CH}_{3}\right)$. ${ }^{13} \mathrm{C}$ NMR $\left(100 \mathrm{MHz}, \mathrm{DMSO}-d_{6}\right): \delta_{\mathrm{C}} 153.6(\mathrm{C}=\mathrm{O}), 144.2\left(\underline{\mathrm{C}}_{\mathrm{Ar}}-\mathrm{CH}_{3}\right), 139.8\left(\mathrm{C}_{\mathrm{Ar}}\right), 137.8\left(\mathrm{C}_{\mathrm{Ar}^{-}}\right.$ $\left.\mathrm{SO}_{2}\right), 131.1,129.3,128.6,128.2\left(4 \mathrm{C}_{\mathrm{Ar}} \mathrm{H}\right), 120.8\left(\mathrm{C}_{\mathrm{Ar}-}-\mathrm{Br}\right), 111-118\left(\mathrm{~m}, \mathrm{CF}_{2}\right), 108.8\left(\mathrm{tt}, J_{\mathrm{CF}} 250.1\right.$ $\left.\mathrm{Hz},{ }^{2} J_{\mathrm{CF}} 29.0 \mathrm{~Hz}, \mathrm{HCF}_{2}\right), 82.1\left(\mathrm{t},{ }^{2} J_{\mathrm{CF}} 27.1 \mathrm{~Hz}, \mathrm{C}-4\right), 65.7(\mathrm{C}-5), 49.7(\mathrm{C}-6), 21.1\left(\mathrm{CH}_{3}\right) .{ }^{19} \mathrm{~F}$ NMR (188 MHz, DMSO- $\left.d_{6}\right): \delta_{\mathrm{F}}-126.62\left(2 \mathrm{~F}, \mathrm{~m}, \mathrm{CF}_{2}\right),-135.22\left(2 \mathrm{~F}, \mathrm{ABd}, J_{\mathrm{AB}} 299.1 \mathrm{~Hz},{ }^{2} J_{\mathrm{FH}}\right.$ $\left.52.0 \mathrm{~Hz}, \mathrm{HCF}_{2}\right)$. MS APCI, $m / z(\%)=527$ and $525(\mathrm{MH}, 100$ and 96). Anal. Calcd for $\mathrm{C}_{19} \mathrm{H}_{17} \mathrm{BrF}_{4} \mathrm{~N}_{2} \mathrm{O}_{4} \mathrm{~S}$ (525.31): C, 43.44; H, 3.26; N, 5.33; S, 6.10. Found C, 43.36; H, 3.30; N, 5.42; S, 6.17.

\section{6-Phenyl-4-hydroxy-5-[(4-methylphenyl)sulfonyl]-4-(trifluoromethyl)tetrahydropyrimi-} dine-2(1H)-thione (2g). Colorless solid, yield 56\%, $241 \mathrm{mg}, \mathrm{mp} 188-190{ }^{\circ} \mathrm{C}$ (from $\mathrm{CH}_{3} \mathrm{CN}$ ). ${ }^{1} \mathrm{H}$ NMR (300 MHz, DMSO- $\left.d_{6}\right): \delta_{\mathrm{H}} 9.57(1 \mathrm{H}, \mathrm{d}, J 3.7 \mathrm{~Hz}, \mathrm{NH}-1), 8.12,7.87(2 \mathrm{H}, \mathrm{s}, \mathrm{OH}+\mathrm{NH}-1)$, 7.43 and $7.92\left(4 \mathrm{H}\right.$, dd, $J 7.8 \mathrm{~Hz}, \mathrm{C}_{6} \mathrm{H}_{4}$-para $), 7.27$ and $7.37\left(5 \mathrm{H}, \mathrm{m}, \mathrm{C}_{6} \mathrm{H}_{5}\right), 5.56(1 \mathrm{H}, \mathrm{d}, J 3.7 \mathrm{~Hz}$, $\mathrm{H}-6), 4.31(1 \mathrm{H}, \mathrm{s}, \mathrm{H}-5), 2.42\left(3 \mathrm{H}, \mathrm{s}, \mathrm{CH}_{3}\right) .{ }^{13} \mathrm{C}$ NMR (100 MHz, DMSO- $\left.d_{6}\right): \delta_{\mathrm{C}} 177.5(\mathrm{C}=\mathrm{S})$, $144.3\left(\underline{\mathrm{C}}_{\mathrm{Ar}}-\mathrm{CH}_{3}\right), 139.0\left(\mathrm{C}_{\mathrm{Ar}}-i p s o\right), 138.0\left(\mathrm{C}_{\mathrm{Ar}}-\mathrm{SO}_{2}\right), 129.4,128.7,128.5,127.5,125.5\left(5 \mathrm{C}_{\mathrm{Ar}}-\mathrm{H}\right)$, $122.0\left(\mathrm{q}, J_{\mathrm{CF}} 290.0 \mathrm{~Hz}, \mathrm{CF}_{3}\right), 79.3\left(\mathrm{q},{ }^{2} J_{\mathrm{CF}} 33.0 \mathrm{~Hz}, \mathrm{C}-4\right), 63.6(\mathrm{C}-5), 50.3(\mathrm{C}-6), 21.2\left(\mathrm{CH}_{3}\right) .{ }^{19} \mathrm{~F}$ NMR $\left(188 \mathrm{MHz}, \mathrm{DMSO}-d_{6}\right): \delta_{\mathrm{F}}-81.97\left(3 \mathrm{~F}, \mathrm{~s}, \mathrm{CF}_{3}\right)$. MS APCI, $m / z(\%)=431(\mathrm{MH}, 100)$. Anal. 
Calcd for $\mathrm{C}_{18} \mathrm{H}_{17} \mathrm{~F}_{3} \mathrm{~N}_{2} \mathrm{O}_{3} \mathrm{~S}_{2}$ (430.46): C, 50.22; H, 3.98; N, 6.51; S, 14.90\%. Found C, 50.27; H, $3.90 ; \mathrm{N}, 6.71 ; \mathrm{S}, 14.85 \%$.

4-Hydroxy-6-(4-methoxyphenyl)-5-[(4-methylphenyl)sulfonyl]-4-(trifluoromethyl)tetrahydropyrimidine-2(1H)-thione (2h). Colorless solid, yield 60\%, $276 \mathrm{mg}, \mathrm{mp} 209-211{ }^{\circ} \mathrm{C}$ (from $\mathrm{CH}_{3} \mathrm{CN}$ ). ${ }^{1} \mathrm{H}$ NMR (300 MHz, DMSO- $\left.d_{6}\right): \delta_{\mathrm{H}} 9.28,8.78,7.77(3 \mathrm{H}, \mathrm{s}, 2 \mathrm{NH}, \mathrm{OH}), 7.16$ and 7.39 $\left(4 \mathrm{H}, \mathrm{dd}, J 7.9 \mathrm{~Hz}, \mathrm{C}_{6} \mathrm{H}_{4}-\right.$ para $), 6.58$ and $6.82\left(4 \mathrm{H}, \mathrm{dd}, J 8.4 \mathrm{~Hz}, \mathrm{C}_{6} \mathrm{H}_{4}-\right.$ para $), 4.70(1 \mathrm{H}, \mathrm{d}, J 8.4$ $\mathrm{Hz}, \mathrm{H}-6), 4.39(1 \mathrm{H}, \mathrm{d}, J 8.4 \mathrm{~Hz}, \mathrm{H}-5), 3.67\left(3 \mathrm{H}, \mathrm{s}, \mathrm{OCH}_{3}\right), 2.32\left(3 \mathrm{H}, \mathrm{s}, \mathrm{CH}_{3}\right) .{ }^{13} \mathrm{C} \mathrm{NMR}(100$ MHz, DMSO- $\left.d_{6}\right): \delta_{\mathrm{C}} 175.5(\mathrm{C}=\mathrm{S}), 159.1\left(\underline{\mathrm{C}}_{\mathrm{Ar}}-\mathrm{OCH}_{3}\right), 144.6\left(\underline{\mathrm{C}}_{\mathrm{Ar}}-\mathrm{CH}_{3}\right), 137.6\left(\mathrm{C}_{\mathrm{Ar}}-\mathrm{SO}_{2}\right), 132.0$ $\left(\mathrm{C}_{\mathrm{Ar}}\right), 129.2,129.1,127.3\left(3 \mathrm{C}_{\mathrm{Ar}}-\mathrm{H}\right), 128.8\left(\mathrm{C}_{\mathrm{Ar}}\right), 122.4\left(\mathrm{q}, J_{\mathrm{CF}} 288.0 \mathrm{~Hz}, \mathrm{CF}_{3}\right), 113.5\left(\mathrm{C}_{\mathrm{Ar}}\right), 81.0$ $\left(\mathrm{t},{ }^{2} J_{\mathrm{CF}} 26.9 \mathrm{~Hz}, \mathrm{C}-4\right), 64.9(\mathrm{C}-5), 55.1\left(\mathrm{OCH}_{3}\right), 54.9(\mathrm{C}-6), 21.0\left(\mathrm{CH}_{3}\right) .{ }^{19} \mathrm{~F}$ NMR $(188 \mathrm{MHz}$, DMSO- $\left.d_{6}\right): \delta_{\mathrm{F}}-76.19\left(3 \mathrm{~F}, \mathrm{~s}, \mathrm{CF}_{3}\right)$. MS APCI, $\mathrm{m} / z(\%)=461(\mathrm{MH}, 100)$. Anal. Calcd for $\mathrm{C}_{19} \mathrm{H}_{19} \mathrm{~F}_{3} \mathrm{~N}_{2} \mathrm{O}_{4} \mathrm{~S}_{2}$ (460.49): C, 49.56; H, 4.16; N, 6.08; S, 13.93\%. Found C, 49.50; H, 4.25; N, $6.20 ; \mathrm{S}, 13.99 \%$.

6-(4-Bromophenyl)-4-hydroxy-5-[(4-methylphenyl)sulfonyl]-4-(trifluoromethyl)tetrahydro-

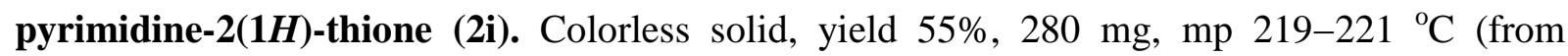
EtOH). ${ }^{1} \mathrm{H}$ NMR $\left(300 \mathrm{MHz}, \mathrm{DMSO}-d_{6}\right): \delta_{\mathrm{H}} 9.57(1 \mathrm{H}, \mathrm{d}, J 3.5 \mathrm{~Hz}, \mathrm{NH}-1), 8.28,7.92(2 \mathrm{H}, \mathrm{s}, \mathrm{OH}$ $+\mathrm{NH}-3), 7.41$ and $7.89\left(4 \mathrm{H}, \mathrm{dd}, J 8.0 \mathrm{~Hz}, \mathrm{C}_{6} \mathrm{H}_{4}\right.$-para), 7.25 and $7.56\left(4 \mathrm{H}, \mathrm{dd}, J 8.4 \mathrm{~Hz}, \mathrm{C}_{6} \mathrm{H}_{4}-\right.$ para), $5.48(1 \mathrm{H}, \mathrm{d}, J 3.5 \mathrm{~Hz}, \mathrm{H}-6), 4.36(1 \mathrm{H}, \mathrm{s}, \mathrm{H}-5), 2.41\left(3 \mathrm{H}, \mathrm{s}, \mathrm{CH}_{3}\right) .{ }^{13} \mathrm{C} \mathrm{NMR}(100 \mathrm{MHz}$, DMSO- $\left.d_{6}\right): \delta_{\mathrm{C}} 177.4(\mathrm{C}=\mathrm{S}), 144.1\left(\underline{\mathrm{C}}_{\mathrm{Ar}}-\mathrm{CH}_{3}\right), 138.1\left(\mathrm{C}_{\mathrm{Ar}}\right), 137.8\left(\mathrm{C}_{\mathrm{Ar}}-\mathrm{SO}_{2}\right), 131.1,129.2,128.5$, $127.9\left(4 \mathrm{C}_{\mathrm{Ar}}-\mathrm{H}\right), 121.8\left(\mathrm{q}, J_{\mathrm{CF}} 293.0 \mathrm{~Hz}, \mathrm{CF}_{3}\right), 120.7\left(\mathrm{C}_{\mathrm{Ar}}-\mathrm{Br}\right), 79.4\left(\mathrm{q},{ }^{2} J_{\mathrm{CF}} 33.0 \mathrm{~Hz}, \mathrm{C}-4\right), 63.3$ (C-5), $50.2(\mathrm{C}-6), 21.0\left(\mathrm{CH}_{3}\right) .{ }^{19} \mathrm{~F}$ NMR (188 MHz, DMSO- $\left.d_{6}\right): \delta_{\mathrm{F}}-81.45\left(3 \mathrm{~F}, \mathrm{~s}, \mathrm{CF}_{3}\right) . \mathrm{MS}$ APCI, $m / z(\%)=511$ and $509\left(\mathrm{MH}, 100\right.$ and 94). Anal. Calcd for $\mathrm{C}_{18} \mathrm{H}_{16} \mathrm{BrF}_{3} \mathrm{~N}_{2} \mathrm{O}_{3} \mathrm{~S}_{2}$ (509.36): C, 42.44; H, 3.17; N, 5.50; S 12.59\%. Found C, 42.40; H, 3.24; N, 5.61; S 12.65\%.

4-Hydroxy-5-[(4-methylphenyl)sulfonyl]-6-phenyl-4-(1,1,2,2-tetrafluoroethyl)tetrahydropyrimidin-2(1H)-thione (2j). Colorless solid, yield 63\%, $291 \mathrm{mg}, \mathrm{mp} \mathrm{185-186}{ }^{\circ} \mathrm{C}$ (from $\mathrm{CH}_{3} \mathrm{CN}$ ). ${ }^{1} \mathrm{H}$ NMR $\left(300 \mathrm{MHz}, \mathrm{DMSO}-d_{6}\right): \delta_{\mathrm{H}} 9.55(1 \mathrm{H}, \mathrm{d}, J 4.0 \mathrm{~Hz}, \mathrm{NH}-1), 7.95,7.80(2 \mathrm{H}, \mathrm{s}, \mathrm{OH}+\mathrm{NH}-$ 1), 7.44 and $7.90\left(4 \mathrm{H}, \mathrm{dd}, J 8.2 \mathrm{~Hz}, \mathrm{C}_{6} \mathrm{H}_{4}\right.$-para $), 7.22-7.38\left(5 \mathrm{H}, \mathrm{m}, \mathrm{C}_{6} \mathrm{H}_{5}\right), 6.34\left(1 \mathrm{H}, \mathrm{tt},{ }^{2} J_{\mathrm{HF}} 51.8\right.$ $\left.\mathrm{Hz},{ }^{3} J_{\mathrm{HF}} 6.3 \mathrm{~Hz}, \mathrm{HCF}_{2}\right), 5.57(1 \mathrm{H}, \mathrm{d}, J 4.0 \mathrm{~Hz}, \mathrm{H}-6), 4.35(1 \mathrm{H}, \mathrm{s}, \mathrm{H}-5), 2.41\left(3 \mathrm{H}, \mathrm{s}, \mathrm{CH}_{3}\right) .{ }^{13} \mathrm{C}$ NMR $\left(100 \mathrm{MHz}, \mathrm{DMSO}-d_{6}\right): \delta_{\mathrm{C}} 177.2(\mathrm{C}=\mathrm{S}), 144.2\left(\underline{\mathrm{C}}_{\mathrm{Ar}}-\mathrm{CH}_{3}\right), 138.9\left(\mathrm{C}_{\mathrm{Ph}^{-}}-i p s o\right), 137.7\left(\mathrm{C}_{\mathrm{Ar}^{-}}\right.$ $\left.\mathrm{SO}_{2}\right), 129.4,128.5,128.2,127.3,125.4\left(5 \mathrm{C}_{\mathrm{Ar}} \mathrm{H}\right), 111-118\left(\mathrm{~m}, \mathrm{CF}_{2}\right), 108.6\left(\mathrm{tt}, J_{\mathrm{CF}} 250.9 \mathrm{~Hz},{ }^{2} J_{\mathrm{CF}}\right.$ $\left.31.5 \mathrm{~Hz}, \mathrm{HCF}_{2}\right), 80.4\left(\mathrm{t},{ }^{2} J_{\mathrm{CF}}=27.0 \mathrm{~Hz}, \mathrm{C}-4\right), 63.8(\mathrm{C}-5), 50.5(\mathrm{C}-6), 21.0\left(\mathrm{CH}_{3}\right) .{ }^{19} \mathrm{~F} \mathrm{NMR}(188$ $\left.\mathrm{MHz}, \mathrm{DMSO}-d_{6}\right): \delta_{\mathrm{F}}-126.04\left(2 \mathrm{~F}, \mathrm{~m}, \mathrm{CF}_{2}\right),-135.67\left(2 \mathrm{~F}, \mathrm{dm},{ }^{2} J_{\mathrm{FH}} 51.8 \mathrm{~Hz}, \mathrm{HCF}_{2}\right)$. MS APCI, $m / z(\%)=463(\mathrm{MH}, 100)$. Anal. Calcd for $\mathrm{C}_{19} \mathrm{H}_{18} \mathrm{~F}_{4} \mathrm{~N}_{2} \mathrm{O}_{3} \mathrm{~S}_{2}$ (462.48): C, 49.34; H, 3.92; N, 6.06; $\mathrm{S}, 13.87 \%$. Found C, 49.21; H, 3.99; N, 6.14; S, 13.99\%.

4-Hydroxy-6-(4-methoxyphenyl)-5-[(4-methylphenyl)sulfonyl]-4-(1,1,2,2-tetrafluoroethyl)tetrahydropyrimidin-2(1H)-thione (2k). Colorless solid, yield 53\%, $261 \mathrm{mg}, \mathrm{mp} 182-183{ }^{\circ} \mathrm{C}$ (from $\mathrm{CH}_{3} \mathrm{CN}$ ). ${ }^{1} \mathrm{H}$ NMR (300 MHz, DMSO- $\left.d_{6}\right)$ : $\delta_{\mathrm{H}} 9.45(1 \mathrm{H}, \mathrm{d}, J 3.1 \mathrm{~Hz}, \mathrm{NH}-1), 7.88,7.67$ $(2 \mathrm{H}, \mathrm{s}, \mathrm{NH}+\mathrm{OH}), 7.37$ and $7.80\left(4 \mathrm{H}, \mathrm{dd}, J 8.1 \mathrm{~Hz}, \mathrm{C}_{6} \mathrm{H}_{4}-\right.$ para $), 6.84$ and $7.07(4 \mathrm{H}, \mathrm{dd}, J 8.1 \mathrm{~Hz}$, $\mathrm{C}_{6} \mathrm{H}_{4}$-para $), 6.32\left(1 \mathrm{H}, \mathrm{tt},{ }^{2} J_{\mathrm{HF}} 52.0 \mathrm{~Hz},{ }^{3} J_{\mathrm{HF}} 6.2 \mathrm{~Hz}, \mathrm{HCF}_{2}\right), 5.43(1 \mathrm{H}, \mathrm{d}, J 3.1 \mathrm{~Hz}, \mathrm{H}-6), 4.27$ $(1 \mathrm{H}, \mathrm{s}, \mathrm{H}-5), 3.82\left(3 \mathrm{H}, \mathrm{s}, \mathrm{OCH}_{3}\right), 2.41\left(3 \mathrm{H}, \mathrm{s}, \mathrm{CH}_{3}\right) .{ }^{13} \mathrm{C} \mathrm{NMR}\left(100 \mathrm{MHz}, \mathrm{DMSO}-d_{6}\right): \delta_{\mathrm{C}} 177.2$ 
$(\mathrm{C}=\mathrm{S}), 158.6\left(\underline{\mathrm{C}}_{\mathrm{Ar}}-\mathrm{OCH}_{3}\right), 144.2\left(\underline{\mathrm{C}}_{\mathrm{Ar}}-\mathrm{CH}_{3}\right), 137.9\left(\mathrm{C}_{\mathrm{Ar}}-\mathrm{SO}_{2}\right), 132.5\left(\mathrm{C}_{\mathrm{Ar}}\right), 129.5,128.5,126.1$ $\left(3 \mathrm{C}_{\mathrm{Ar}} \mathrm{H}\right), 113.7\left(\mathrm{C}_{\mathrm{Ar}}\right), 113.4\left(\mathrm{~m}, \mathrm{CF}_{2}\right), 108.8\left(\mathrm{tt}, J_{\mathrm{CF}} 251.0 \mathrm{~Hz},{ }^{2} J_{\mathrm{CF}} 30.1 \mathrm{~Hz}, \mathrm{HCF}_{2}\right), 80.6\left(\mathrm{t},{ }^{2} J_{\mathrm{CF}}\right.$ $27.5 \mathrm{~Hz}, \mathrm{C}-4), 64.0(\mathrm{C}-5), 55.1\left(\mathrm{OCH}_{3}\right), 50.3(\mathrm{C}-6), 21.1\left(\mathrm{CH}_{3}\right) .{ }^{19} \mathrm{~F}$ NMR (188 MHz, DMSO$\left.d_{6}\right): \delta_{\mathrm{F}}-125.70\left(2 \mathrm{~F}, \mathrm{ABm}, J_{\mathrm{AB}} 262.8 \mathrm{~Hz}, \mathrm{CF}_{2}\right),-135.52\left(1 \mathrm{~F}, \mathrm{dm},{ }^{2} J_{\mathrm{FH}} 52.0 \mathrm{~Hz}, \mathrm{HCF}_{2}\right) . \mathrm{MS}$ APCI, $m / z(\%)=493(\mathrm{MH}, 100)$. Anal. Calcd for $\mathrm{C}_{19} \mathrm{H}_{17} \mathrm{BrF}_{4} \mathrm{~N}_{2} \mathrm{O}_{4} \mathrm{~S}$ (492.51): C, 48.77; H, 4.09; N, 5.69; S, 13.02\%. Found C, 48.84; H, 4.18; N, 5.77; S, 13.14\%.

6-(4-Bromophenyl)-4-hydroxy-5-[(4-methylphenyl)sulfonyl]-4-(1,1,2,2-tetrafluoroethyl)tetrahydropyrimidin-2(1H)-thione (2l). Colorless solid, yield 50\%, $270 \mathrm{mg}, \mathrm{mp} 209-211{ }^{\circ} \mathrm{C}$ (from $\mathrm{CH}_{3} \mathrm{CN}$ ). ${ }^{1} \mathrm{H}$ NMR $\left(300 \mathrm{MHz}, \mathrm{DMSO}-d_{6}\right)$ : $\delta_{\mathrm{H}} 9.54(1 \mathrm{H}, \mathrm{d}, J 3.7 \mathrm{~Hz}, \mathrm{NH}-1), 8.00,7.88$ $(2 \mathrm{H}, \mathrm{s}, \mathrm{OH}+\mathrm{NH}-3), 7.41$ and $7.87\left(4 \mathrm{H}, \mathrm{dd}, J 8.4 \mathrm{~Hz}, \mathrm{C}_{6} \mathrm{H}_{4}-\right.$ para $), 7.20$ and $7.54(4 \mathrm{H}, \mathrm{dd}, J 8.4$ $\mathrm{Hz}, \mathrm{C}_{6} \mathrm{H}_{4}$-para $), 6.36\left(1 \mathrm{H}, \mathrm{tt},{ }^{2} J_{\mathrm{HF}} 52.8 \mathrm{~Hz},{ }^{3} J_{\mathrm{HF}} 6.2 \mathrm{~Hz}, \mathrm{HCF}_{2}\right), 5.50(1 \mathrm{H}, \mathrm{d}, J 3.7 \mathrm{~Hz}, \mathrm{H}-6), 4.36$ $(1 \mathrm{H}, \mathrm{s}, \mathrm{H}-5), 2.43\left(3 \mathrm{H}, \mathrm{s}, \mathrm{CH}_{3}\right) .{ }^{13} \mathrm{C}$ NMR $\left(100 \mathrm{MHz}, \mathrm{DMSO}-d_{6}\right): \delta_{\mathrm{C}} 177.2(\mathrm{C}=\mathrm{S}), 144.4\left(\underline{\mathrm{C}}_{\mathrm{Ar}^{-}}\right.$ $\left.\mathrm{CH}_{3}\right), 138.4\left(\mathrm{C}_{\mathrm{Ar}}\right), 137.8\left(\mathrm{C}_{\mathrm{Ar}}-\mathrm{SO}_{2}\right), 131.3,129.6,128.6,128.1\left(4 \mathrm{C}_{\mathrm{Ar}}-\mathrm{H}\right), 120.8\left(\mathrm{C}_{\mathrm{Ar}} \mathrm{Br}\right), 111-$ $118\left(\mathrm{~m}, \mathrm{CF}_{2}\right), 108.6\left(\mathrm{tt}, J_{\mathrm{CF}} 250.9 \mathrm{~Hz},{ }^{2} J_{\mathrm{CF}} 30.1 \mathrm{~Hz}, \mathrm{HCF}_{2}\right), 80.6\left(\mathrm{t},{ }^{2} J_{\mathrm{CF}} 27.9 \mathrm{~Hz}, \mathrm{C}-4\right), 63.4(\mathrm{C}-$ 5), $50.3(\mathrm{C}-6), 21.2\left(\mathrm{CH}_{3}\right) .{ }^{19} \mathrm{~F}$ NMR (188 MHz, DMSO- $\left.d_{6}\right): \delta_{\mathrm{F}}-125.43\left(2 \mathrm{~F}, \mathrm{~m}, \mathrm{CF}_{2}\right),-135.63$ $\left(2 \mathrm{~F}, \mathrm{dm},{ }^{2} J_{\mathrm{FH}} 52.8 \mathrm{~Hz}, \mathrm{HCF}_{2}\right)$. MS APCI, $m / z(\%)=543$ and $541(\mathrm{MH}, 100$ and 93). Anal. Calcd for $\mathrm{C}_{19} \mathrm{H}_{17} \mathrm{BrF}_{4} \mathrm{~N}_{2} \mathrm{O}_{3} \mathrm{~S}_{2}$ (541.38): C, 42.15; H, 3.17; N, 5.17; $\mathrm{S} 11.85 \%$. Found $\mathrm{C}, 42.20 ; \mathrm{H}, 3.10$; $\mathrm{N}, 5.11 ; \mathrm{S}, 11.83 \%$.

$\mathrm{N}, \mathrm{N}$-Diethyl-4-hydroxy-2-oxo-6-phenyl-4-(trifluoromethyl)-hexahydropyrimidine-5-sulfonamide (2m). Colorless needles, yield 82\%, $324 \mathrm{mg}, \mathrm{mp} 192-194{ }^{\circ} \mathrm{C}$ (from EtOH- $\left.\mathrm{H}_{2} \mathrm{O}\right)$; IR ( $v_{\max }$, $\left.\mathrm{cm}^{-1}\right): 3370,3230,3120,2990(\mathrm{NH}, \mathrm{OH}), 1700(\mathrm{C}=\mathrm{O}) .{ }^{1} \mathrm{H}$ NMR $\left(400 \mathrm{MHz}, \mathrm{DMSO}-d_{6}\right): \delta_{\mathrm{H}} 7.55$ $(2 \mathrm{H}, \mathrm{m}, \mathrm{NH}-1+\mathrm{OH}), 7.23-7.39\left(5 \mathrm{H}, \mathrm{m}, \mathrm{C}_{6} \mathrm{H}_{5}\right), 6.88(1 \mathrm{H}, \mathrm{s}, \mathrm{NH}-3), 5.23(1 \mathrm{H}, \mathrm{d}, J 3.0 \mathrm{~Hz}, \mathrm{H}-6)$, $3.89(1 \mathrm{H}, \mathrm{s}, \mathrm{H}-5), 3.17-3.39\left(4 \mathrm{H}, \mathrm{m}, 2 \mathrm{CH}_{2}\right), 1.12\left(6 \mathrm{H}, \mathrm{t}, J 6.9 \mathrm{~Hz}, 2 \mathrm{CH}_{3}\right) .{ }^{13} \mathrm{C} \mathrm{NMR}(100 \mathrm{MHz}$, DMSO- $\left.d_{6}\right): \delta_{\mathrm{C}} 154.2(\mathrm{C}=\mathrm{O}), 141.0\left(\mathrm{C}_{\mathrm{Ph}}-\right.$ ipso $), 128.4,125.6\left(2 \mathrm{C}_{\mathrm{Ph}}-\mathrm{H}\right), 127.2\left(\mathrm{C}_{\mathrm{Ph}}-\right.$ para $), 122.8(\mathrm{q}$, $\left.J_{\mathrm{CF}} 285.6 \mathrm{~Hz}, \mathrm{CF}_{3}\right), 81.0\left(\mathrm{q},{ }^{2} J_{\mathrm{CF}} 32.3 \mathrm{~Hz}, \mathrm{C}-4\right), 63.7(\mathrm{C}-5), 52.1(\mathrm{C}-6), 41.9\left(2 \mathrm{CH}_{2}\right), 14.6$ $\left(2 \mathrm{CH}_{3}\right) .{ }^{19} \mathrm{~F}$ NMR $\left(188 \mathrm{MHz}, \mathrm{DMSO}-d_{6}\right): \delta_{\mathrm{F}}-82.51\left(3 \mathrm{~F}, \mathrm{~s}, \mathrm{CF}_{3}\right)$. MS APCI, $m / z(\%)=396(\mathrm{MH}$, 100). Anal. Calcd for $\mathrm{C}_{15} \mathrm{H}_{20} \mathrm{~F}_{3} \mathrm{~N}_{3} \mathrm{O}_{4} \mathrm{~S}$ (395.40): $\mathrm{C}, 45.56 ; \mathrm{H}, 5.10 ; \mathrm{N}, 10.63 ; \mathrm{S}, 8.11 \%$. Found: $\mathrm{C}$, 45.50; H, 5.12; N, 10.69; S, 8.15\%.

$N, N$-Diethyl-4-hydroxy-2-oxo-6-(4-methoxyphenyl)-4-(trifluoromethyl)-hexahydropyrimidine-5-sulfonamide (2n). Colorless needles, yield 83\%, $353 \mathrm{mg}, \mathrm{mp} 179-181{ }^{\circ} \mathrm{C}$ (from EtOH$\left.\mathrm{H}_{2} \mathrm{O}\right)$; IR $\left(v_{\max }, \mathrm{cm}^{-1}\right): 3360,3230,3110,2980(\mathrm{NH}, \mathrm{OH}), 1690(\mathrm{C}=\mathrm{O}) .{ }^{1} \mathrm{H}$ NMR $(400 \mathrm{MHz}$, DMSO- $\left.d_{6}\right): \delta_{\mathrm{H}} 7.50(2 \mathrm{H}, \mathrm{m}, \mathrm{NH}-1+\mathrm{OH}), 6.91-7.17\left(4 \mathrm{H}, \mathrm{dd}, J 8.4 \mathrm{~Hz}, \mathrm{C}_{6} \mathrm{H}_{4}-\right.$ para $), 6.86(1 \mathrm{H}, \mathrm{s}$, NH-3), 5.16 (1H, m, H-6), $3.84(1 \mathrm{H}, \mathrm{s}, \mathrm{H}-5), 3.73\left(3 \mathrm{H}, \mathrm{s}, \mathrm{OCH}_{3}\right), 3.14-3.36\left(4 \mathrm{H}, \mathrm{m}, 2 \mathrm{CH}_{2}\right), 1.11$ $\left(6 \mathrm{H}, \mathrm{t}, J 6.9 \mathrm{~Hz}, 2 \mathrm{CH}_{3}\right) .{ }^{13} \mathrm{C} \mathrm{NMR}\left(100 \mathrm{MHz}, \mathrm{DMSO}-d_{6}\right): \delta_{\mathrm{C}} 158.4\left(\underline{\mathrm{C}}_{\mathrm{Ar}}-\mathrm{OCH}_{3}\right), 154.1(\mathrm{C}=\mathrm{O})$, $132.7\left(\mathrm{C}_{\mathrm{Ar}}\right), 126.8\left(\mathrm{C}_{\mathrm{Ar}}\right), 122.8\left(\mathrm{q}, J_{\mathrm{CF}} 288.9 \mathrm{~Hz}, \mathrm{CF}_{3}\right), 113.7\left(\mathrm{C}_{\mathrm{Ar}}\right), 81.0\left(\mathrm{q},{ }^{2} J_{\mathrm{CF}} 32.6 \mathrm{~Hz}, \mathrm{C}-4\right)$, 63.9 (C-5), $55.1\left(\mathrm{OCH}_{3}\right), 51.7(\mathrm{C}-6), 41.8\left(2 \mathrm{CH}_{2}\right), 14.6\left(2 \mathrm{CH}_{3}\right) .{ }^{19} \mathrm{~F}$ NMR $(188 \mathrm{MHz}, \mathrm{DMSO}-$ $\left.d_{6}\right): \delta_{\mathrm{F}}-82.30\left(3 \mathrm{~F}, \mathrm{~s}, \mathrm{CF}_{3}\right)$. MS APCI, $m / z(\%)=426(\mathrm{MH}, 100)$. Anal. Calcd for $\mathrm{C}_{16} \mathrm{H}_{22} \mathrm{~F}_{3} \mathrm{~N}_{3} \mathrm{O}_{5} \mathrm{~S}$ (425.42): C, 45.17; H, 5.21; N, 9.88; S, 7.54\%. Found: C, 45.15; H, 5.25; N, 10.00; S, 7.60\%.

$\mathrm{N}, \mathrm{N}$-Diethyl-4-hydroxy-2-oxo-6-phenyl-4-(1,1,2,2-tetrafluoroethyl)-hexahydropyrimidine-

5-sulfonamide (2o). Colorless solid, yield 78\%, $333 \mathrm{mg}, \mathrm{mp} 184-185{ }^{\circ} \mathrm{C}$ (from EtOH- $\mathrm{H}_{2} \mathrm{O}$ ); IR 
$\left(v_{\max }, \mathrm{cm}^{-1}\right): 3370,3240,3120,2990(\mathrm{NH}, \mathrm{OH}), 1690(\mathrm{C}=\mathrm{O}) .{ }^{1} \mathrm{H}$ NMR $\left(400 \mathrm{MHz}, \mathrm{DMSO}-d_{6}\right)$ : $\delta_{\mathrm{H}} 7.58(1 \mathrm{H}, \mathrm{d}, J 3.0 \mathrm{~Hz}, \mathrm{NH}-1), 7.49(1 \mathrm{H}, \mathrm{s}, \mathrm{OH}), 7.20-7.37\left(5 \mathrm{H}, \mathrm{m}, \mathrm{C}_{6} \mathrm{H}_{5}\right), 6.60(1 \mathrm{H}, \mathrm{s}, \mathrm{NH}-3)$, $6.48\left(1 \mathrm{H}, \mathrm{tt},{ }^{2} J_{\mathrm{HF}} 52.0 \mathrm{~Hz},{ }^{3} J_{\mathrm{HF}} 5.4 \mathrm{~Hz}, \mathrm{HCF}_{2}\right), 5.19(1 \mathrm{H}, \mathrm{m}, \mathrm{H}-6), 4.00(1 \mathrm{H}, \mathrm{s}, \mathrm{H}-5), 3.20-3.33$ $\left(4 \mathrm{H}, \mathrm{m}, 2 \mathrm{CH}_{2}\right), 1.12\left(6 \mathrm{H}, \mathrm{t}, J 6.9 \mathrm{~Hz}, 2 \mathrm{CH}_{3}\right) .{ }^{13} \mathrm{C}$ NMR $\left(100 \mathrm{MHz}, \mathrm{DMSO}-d_{6}\right): \delta_{\mathrm{C}} 154.4(\mathrm{C}=\mathrm{O})$, $141.0\left(\mathrm{C}_{\mathrm{Ph}}\right.$-ipso $), 128.4,125.7\left(2 \mathrm{C}_{\mathrm{Ar}}-\mathrm{H}\right), 127.3\left(\mathrm{C}_{\mathrm{Ph}}\right.$-para $), 111-118\left(\mathrm{~m}, \mathrm{CF}_{2}\right), 109.0\left(\mathrm{tt}, J_{\mathrm{CF}} 249.2\right.$ $\left.\mathrm{Hz},{ }^{2} J_{\mathrm{CF}} 28.6 \mathrm{~Hz}, \mathrm{HCF}_{2}\right), 82.1\left(\mathrm{t},{ }^{2} J_{\mathrm{CF}} 27.1 \mathrm{~Hz}, \mathrm{C}-4\right), 63.6(\mathrm{C}-5), 52.1(\mathrm{C}-6), 41.9\left(2 \mathrm{CH}_{2}\right), 14.7$ $\left(2 \mathrm{CH}_{3}\right) .{ }^{19} \mathrm{~F}$ NMR $\left(188 \mathrm{MHz}, \mathrm{DMSO}-d_{6}\right): \delta_{\mathrm{F}} \delta_{\mathrm{A}}-126.48\left(1 \mathrm{~F}, \mathrm{AB}, J_{\mathrm{AB}} 259.5 \mathrm{~Hz}, \mathrm{CF}_{\mathrm{A}} \mathrm{F}_{\mathrm{B}}\right), \delta_{\mathrm{B}}-$ $127.23\left(1 \mathrm{~F}, \mathrm{AB}, J_{\mathrm{AB}} 259.5 \mathrm{~Hz}, \mathrm{CF}_{\mathrm{A}} \underline{\mathrm{F}}_{\mathrm{B}}\right), \delta_{\mathrm{A}}-134.22\left(1 \mathrm{~F}, \mathrm{ABd}, J_{\mathrm{AB}} 297.0 \mathrm{~Hz},{ }^{2} J_{\mathrm{FH}} 52.0 \mathrm{~Hz}\right.$, $\left.\mathrm{HCF}_{\mathrm{A}} \mathrm{F}_{\mathrm{B}}\right), \delta_{\mathrm{B}}-136.11\left(1 \mathrm{~F}, \mathrm{ABd}, J_{\mathrm{AB}} 297.0 \mathrm{~Hz},{ }^{2} J_{\mathrm{FH}} 52.0 \mathrm{~Hz}, \mathrm{HCF}_{\mathrm{A}} \underline{\mathrm{F}}_{\mathrm{B}}\right) . \mathrm{MS}$ APCI, $m / z(\%)=428$ (MH, 100). Anal. Calcd for $\mathrm{C}_{16} \mathrm{H}_{21} \mathrm{~F}_{4} \mathrm{~N}_{3} \mathrm{O}_{4} \mathrm{~S}$ (427.41): C, 44.96; H, 4.95; N, 9.83; S, 7.50\%. Found: C, 45.16; H, 5.12; N, 9.90; S, 7.33\%.

$\mathrm{N}, \mathrm{N}$-Diethyl-4-hydroxy-2-oxo-6-(4-methoxyphenyl)-4-(1,1,2,2-tetrafluoroethyl)-hexahydropyrimidine-5-sulfonamide (2p). Colorless solid, yield 75\%, $343 \mathrm{mg}, \mathrm{mp} 185-187{ }^{\circ} \mathrm{C}$ (from EtOH-H $\left.{ }_{2} \mathrm{O}\right)$; IR $\left(v_{\max }, \mathrm{cm}^{-1}\right): 3355,3230,3115,2990(\mathrm{NH}, \mathrm{OH}), 1685(\mathrm{C}=\mathrm{O}) .{ }^{1} \mathrm{H}$ NMR $(300$ $\left.\mathrm{MHz}, \mathrm{DMSO}-d_{6}\right): \delta_{\mathrm{H}} 7.51(1 \mathrm{H}, \mathrm{m}, \mathrm{NH}-1), 7.45(\mathrm{~s}, 1 \mathrm{H}, \mathrm{OH}), 6.91$ and $7.15(4 \mathrm{H}, \mathrm{dd}, J 8.4 \mathrm{~Hz}$, $\mathrm{C}_{6} \mathrm{H}_{4}$-para), $6.55(\mathrm{~s}, 1 \mathrm{H}, \mathrm{NH}-3), 6.50\left(1 \mathrm{H}, \mathrm{tt},{ }^{2} J_{\mathrm{HF}} 52.0 \mathrm{~Hz},{ }^{3} J_{\mathrm{HF}} 6.6 \mathrm{~Hz}, \mathrm{HCF}_{2}\right), 5.11(1 \mathrm{H}, \mathrm{m}, \mathrm{H}-$ 6), $3.95(1 \mathrm{H}, \mathrm{m}, \mathrm{H}-5), 3.73\left(3 \mathrm{H}, \mathrm{s}, \mathrm{OCH}_{3}\right), 3.20-3.28\left(4 \mathrm{H}, \mathrm{m}, 2 \mathrm{CH}_{2}\right), 1.11(6 \mathrm{H}, \mathrm{t}, J 6.9 \mathrm{~Hz}$, $\left.2 \mathrm{CH}_{3}\right) .{ }^{13} \mathrm{C}$ NMR $\left(100 \mathrm{MHz}, \mathrm{DMSO}-d_{6}\right): \delta_{\mathrm{C}} 158.4\left(\mathrm{C}_{\mathrm{Ar}}-\mathrm{OCH}_{3}\right), 154.2(\mathrm{C}=\mathrm{O}), 132.7\left(\mathrm{C}_{\mathrm{Ar}}\right), 127.0$ $\left(\mathrm{C}_{\mathrm{Ar}}\right), 113.7\left(\mathrm{C}_{\mathrm{Ar}}\right), 111-118\left(\mathrm{~m}, \mathrm{CF}_{2}\right), 106-112\left(\mathrm{~m}, \mathrm{HCF}_{2}\right), 82.1\left(\mathrm{t},{ }^{2} J_{\mathrm{CF}} 27.0 \mathrm{~Hz}, \mathrm{C}-4\right), 63.8(\mathrm{C}-5)$, $55.1\left(\mathrm{OCH}_{3}\right), 51.7(\mathrm{C}-6), 41.8\left(2 \mathrm{CH}_{2}\right), 14.7\left(2 \mathrm{CH}_{3}\right) .{ }^{19} \mathrm{~F}$ NMR $\left(188 \mathrm{MHz}, \mathrm{DMSO}-d_{6}\right): \delta_{\mathrm{F}}-126.63$ $\left(2 \mathrm{~F}, \mathrm{~m}, \mathrm{CF}_{2}\right), \delta_{\mathrm{A}}-134.17\left(1 \mathrm{~F}, \mathrm{ABd}, J_{\mathrm{AB}} 298.6 \mathrm{~Hz},{ }^{2} J_{\mathrm{FH}} 52.0 \mathrm{~Hz}, \mathrm{HCF}_{\mathrm{A}} \mathrm{F}_{\mathrm{B}}\right), \delta_{\mathrm{B}}-135.93(1 \mathrm{~F}, \mathrm{ABd}$, $\left.J_{\mathrm{AB}} 298.6 \mathrm{~Hz},{ }^{2} J_{\mathrm{FH}} 52.0 \mathrm{~Hz}, \mathrm{HCF}_{\mathrm{A}} \underline{\mathrm{F}}_{\mathrm{B}}\right)$. MS APCI, $\mathrm{m} / z(\%)=458(\mathrm{MH}, 100)$. Anal. Calcd for $\mathrm{C}_{17} \mathrm{H}_{23} \mathrm{~F}_{4} \mathrm{~N}_{3} \mathrm{O}_{5} \mathrm{~S}$ (457.44): C, 44.64; H, 5.07; N, 9.19; S, 7.01\%. Found: C, 44.89; H, 5.25; N, 9.29; S, 7.20\%.

$N, N$-Diethyl-4-hydroxy-2-thioxo-6-phenyl-4-(trifluoromethyl)-hexahydropyrimidine-5sulfonamide (2q). Colorless solid, yield $61 \%, 251 \mathrm{mg}, \mathrm{mp} 181-183{ }^{\circ} \mathrm{C}$ (from $\mathrm{CH}_{3} \mathrm{CN}$ ). ${ }^{1} \mathrm{H} \mathrm{NMR}$ (400 MHz, DMSO- $\left.d_{6}\right): \delta_{\mathrm{H}} 9.48(1 \mathrm{H}, \mathrm{d}, J 3.7 \mathrm{~Hz}, \mathrm{NH}-1), 8.21,7.84(2 \mathrm{H}, \mathrm{s}, \mathrm{OH}+\mathrm{NH}-3), 7.23-$ $7.40\left(5 \mathrm{H}, \mathrm{m}, \mathrm{C}_{6} \mathrm{H}_{5}\right), 5.34(1 \mathrm{H}, \mathrm{d}, J 3.7 \mathrm{~Hz}, \mathrm{H}-6), 3.99(1 \mathrm{H}, \mathrm{s}, \mathrm{H}-5), 3.17-3.39\left(4 \mathrm{H}, \mathrm{m}, 2 \mathrm{CH}_{2}\right)$, $1.14\left(6 \mathrm{H}, \mathrm{t}, J 7.2 \mathrm{~Hz}, 2 \mathrm{CH}_{3}\right) .{ }^{13} \mathrm{C}$ NMR (100 MHz, DMSO-d 6$): \delta_{\mathrm{C}} 177.3(\mathrm{C}=\mathrm{S}), 139.3\left(\mathrm{C}_{\mathrm{Ph}}-i p s o\right)$, 128.5, $125.6\left(2 \mathrm{C}_{\mathrm{Ph}}-\mathrm{H}\right), 127.4\left(\mathrm{C}_{\mathrm{Ph}}\right.$-para $), 122.3\left(\mathrm{q}, J_{\mathrm{CF}} 286.5 \mathrm{~Hz}, \mathrm{CF}_{3}\right), 79.4\left(\mathrm{q},{ }^{2} J_{\mathrm{CF}} 33.4 \mathrm{~Hz}, \mathrm{C}-\right.$ 4), $61.4(\mathrm{C}-5), 52.9(\mathrm{C}-6), 41.9\left(2 \mathrm{CH}_{2}\right), 14.6\left(2 \mathrm{CH}_{3}\right) .{ }^{19} \mathrm{~F}$ NMR $\left(188 \mathrm{MHz}, \mathrm{DMSO}-d_{6}\right): \delta_{\mathrm{F}}-81.33$ (3F, s, $\left.\mathrm{CF}_{3}\right)$. MS APCI, $m / z(\%)=412(\mathrm{MH}, 100)$. Anal. Calcd for $\mathrm{C}_{15} \mathrm{H}_{20} \mathrm{~F}_{3} \mathrm{~N}_{3} \mathrm{O}_{3} \mathrm{~S}_{2}$ (411.46): $\mathrm{C}$, 43.79; H, 4.90; N, 10.21; S 15.59\%. Found C, 43.72; H, 4.97; N, 10.33; S 15.60\%.

$N, N$-Diethyl-4-hydroxy-2-thioxo-6-phenyl-4-(1,1,2,2-tetrafluoroethyl)-hexahydropyrimidine-5-sulfonamide (2r). Colorless solid, yield 59\%, $261 \mathrm{mg}, \mathrm{mp} 199-201{ }^{\circ} \mathrm{C}$ (from $\mathrm{CH}_{3} \mathrm{CN}$ ). ${ }^{1} \mathrm{H}$ NMR (400 MHz, DMSO- $\left.d_{6}\right): \delta_{\mathrm{H}} 9.35(1 \mathrm{H}, \mathrm{d}, J 3.6 \mathrm{~Hz}, \mathrm{NH}-1), 7.66,6.95(2 \mathrm{H}, \mathrm{s}, \mathrm{OH}+\mathrm{NH}-$ 3), 7.22-7.39 (5H, m, $\left.\mathrm{C}_{6} \mathrm{H}_{5}\right), 6.49\left(1 \mathrm{H}, \mathrm{tt},{ }^{2} J_{\mathrm{HF}} 52.0 \mathrm{~Hz},{ }^{3} J_{\mathrm{HF}} 6.4 \mathrm{~Hz}, \mathrm{HCF}_{2}\right), 5.34(1 \mathrm{H}, \mathrm{d}, J 3.6$ $\mathrm{Hz}, \mathrm{H}-6), 4.09$ (1H, s, H-5), 3.24-3.40 (4H, m, 2CH $), 1.16\left(6 \mathrm{H}, \mathrm{t}, J 7.1 \mathrm{~Hz}, 2 \mathrm{CH}_{3}\right) .{ }^{13} \mathrm{C} \mathrm{NMR}$ $\left(100 \mathrm{MHz}, \mathrm{DMSO}-d_{6}\right): \delta_{\mathrm{C}} 177.3(\mathrm{C}=\mathrm{S}), 139.2\left(\mathrm{C}_{\mathrm{Ph}}-i p s o\right), 128.1,125.2\left(2 \mathrm{C}_{\mathrm{Ph}^{-}}-\mathrm{H}\right), 127.1\left(\mathrm{C}_{\mathrm{Ph}^{-}}\right.$ para), 111-118 (m, CF $), 108.7$ (tt, $\left.J_{\mathrm{CF}} 250.9 \mathrm{~Hz},{ }^{2} J_{\mathrm{CF}} 30.1 \mathrm{~Hz}, \mathrm{HCF}_{2}\right), 80.2$ (t, ${ }^{2} J_{\mathrm{CF}} 27.0 \mathrm{~Hz}, \mathrm{C}-$ 
4), $61.7(\mathrm{C}-5), 52.9(\mathrm{C}-6), 41.7\left(2 \mathrm{CH}_{2}\right), 14.3\left(2 \mathrm{CH}_{3}\right) .{ }^{19} \mathrm{~F}$ NMR $\left(188 \mathrm{MHz}, \mathrm{DMSO}-d_{6}\right): \delta_{\mathrm{F}}-$ $125.56\left(2 \mathrm{~F}, \mathrm{ABm}, J_{\mathrm{AB}} 258.5 \mathrm{~Hz}, \mathrm{CF}_{2}\right), \delta_{\mathrm{A}}-134.17\left(1 \mathrm{~F}, \mathrm{ABd}, J_{\mathrm{AB}} 298.6 \mathrm{~Hz},{ }^{2} J_{\mathrm{FH}} 52.0 \mathrm{~Hz}\right.$, $\left.\mathrm{HCF}_{\mathrm{A}} \mathrm{F}_{\mathrm{B}}\right), \delta_{\mathrm{B}}-135.93\left(1 \mathrm{~F}, \mathrm{ABd}, J_{\mathrm{AB}} 298.6 \mathrm{~Hz},{ }^{2} J_{\mathrm{FH}} 52.0 \mathrm{~Hz}, \mathrm{HCF}_{\mathrm{A}} \underline{\mathrm{F}}_{\mathrm{B}}\right)$. MS APCI, $m / z(\%)=444$ (MH, 100). Anal. Calcd for $\mathrm{C}_{16} \mathrm{H}_{21} \mathrm{~F}_{4} \mathrm{~N}_{3} \mathrm{O}_{3} \mathrm{~S}_{2}$ (443.48): C, 43.33; H, 4.77; N, 9.48; S, 14.46\%. Found C, 43.52; H, 4.87; N, 9.63; S, 14.60\%.

General synthetic procedure for the three-component reaction of 2-oxo-2polyfluoroalkylethane-1-sulfones (1a,b) and -sulfamide (1e) with urea and trialkyl orthoformate. Compounds 3a-c. A mixture of the corresponding ketosulfone hydrate 1a,b (1 mmol) or ketosulfamide hydrate 1e $(1 \mathrm{mmol})$ and urea $(60 \mathrm{mg}, 1 \mathrm{mmol})$ in trimethyl orthoformate $(6 \mathrm{mmol})$ was refluxed for $3 \mathrm{~h}$. The white solid precipitated from the reaction mixture under heating. The mixture was then allowed to cool, diluted with $\mathrm{Et}_{2} \mathrm{O}(2 \mathrm{~mL})$ and the precipitate was filtered, washed with $\mathrm{Et}_{2} \mathrm{O}$ and dried to give dihydropyrimidinones 3a-c as white solids which were crystallized from an appropriate solvent.

4-Hydroxy-5-tosyl-4-(trifluoromethyl)-3,4-dihydropyrimidin-2(1H)-one (3a). Colorless solid, yield 85\%, $0.29 \mathrm{~g}, \mathrm{mp} 184-186{ }^{\circ} \mathrm{C}$ (from EtOH-H $\left.{ }_{2} \mathrm{O}\right)$; IR $\left(v_{\max }, \mathrm{cm}^{-1}\right)$ : 3370, 3230, 3080, $2910(\mathrm{NH}, \mathrm{OH}), 1710(\mathrm{C}=\mathrm{O}) .{ }^{1} \mathrm{H}$ NMR $\left(300 \mathrm{MHz}, \mathrm{DMSO}-d_{6}\right): \delta_{\mathrm{H}} 10.26(1 \mathrm{H}, \mathrm{d}, J 5.6 \mathrm{~Hz}, \mathrm{NH}-$ 1), 8.57 (1H, s, NH-3), 7.36 and $7.69\left(4 \mathrm{H}, \mathrm{dd}, J 8.1 \mathrm{~Hz}, \mathrm{C}_{6} \mathrm{H}_{4}\right.$-para $), 7.68$ (2H, m, H-6, OH), 2.38 $\left(3 \mathrm{H}, \mathrm{s}, \mathrm{CH}_{3}\right) .{ }^{13} \mathrm{C}$ NMR $\left(100 \mathrm{MHz}, \mathrm{DMSO}-d_{6}\right): \delta_{\mathrm{C}} 149.3(\mathrm{C}=\mathrm{O}), 143.0\left(\underline{\mathrm{C}}_{\mathrm{Ar}}-\mathrm{CH}_{3}\right), 142.0(\mathrm{C}-6)$, $139.8\left(\mathrm{C}_{\mathrm{Ar}}-\mathrm{SO}_{2}\right), 129.1,127.3\left(2 \mathrm{C}_{\mathrm{Ar}} \mathrm{-H}\right), 122.4\left(\mathrm{q}, J_{\mathrm{CF}} 288.7 \mathrm{~Hz}, \mathrm{CF}_{3}\right), 108.2(\mathrm{C}-5), 81.9\left(\mathrm{q},{ }^{2} J_{\mathrm{CF}}\right.$ $33.5 \mathrm{~Hz}, \mathrm{C}-4), 20.8\left(\mathrm{CH}_{3}\right) .{ }^{19} \mathrm{~F}$ NMR $\left(188 \mathrm{MHz}, \mathrm{DMSO}-d_{6}\right): \delta_{\mathrm{F}}-81.49\left(3 \mathrm{~F}, \mathrm{~s}, \mathrm{CF}_{3}\right) . \mathrm{MS}$ APCI, $m / z(\%)=335(\mathrm{M}-\mathrm{H}, 100)$. Anal. Calcd for $\mathrm{C}_{12} \mathrm{H}_{11} \mathrm{~F}_{3} \mathrm{~N}_{2} \mathrm{O}_{4} \mathrm{~S}$ (336.29): C, 42.86; H, 3.30; N, 8.33; S, 9.54\%. Found: C, 42.96; H, 3.40; N, 8.40; S, 9.60\%.

4-Hydroxy-4-(1,1,2,2-tetrafluoroethyl)-5-tosyl-3,4-dihydropyrimidin-2(1H)-one

(3b). Colorless solid, yield 74\%, $0.27 \mathrm{~g}, \mathrm{mp} 254-256{ }^{\circ} \mathrm{C}$ (from $\mathrm{CH}_{3} \mathrm{CN}$ ); IR $\left(v_{\max }, \mathrm{cm}^{-1}\right): 3370,3250$, 3100, $2950(\mathrm{NH}, \mathrm{OH}), 1700(\mathrm{C}=\mathrm{O}) .{ }^{1} \mathrm{H}$ NMR (300 MHz, DMSO- $\left.d_{6}\right): \delta_{\mathrm{H}} 10.21(1 \mathrm{H}, \mathrm{d}, J 5.9 \mathrm{~Hz}$, NH-1), $8.29(1 \mathrm{H}, \mathrm{s}, \mathrm{OH}), 7.36$ and $7.70\left(4 \mathrm{H}, \mathrm{dd}, J 7.2 \mathrm{~Hz}, \mathrm{C}_{6} \mathrm{H}_{4}-\right.$ para $), 7.67(1 \mathrm{H}, \mathrm{d}, J 5.9 \mathrm{~Hz}, \mathrm{H}-$ 6), $7.55(1 \mathrm{H}, \mathrm{s}, \mathrm{NH}-3), 6.56\left(1 \mathrm{H}, \mathrm{tt},{ }^{2} J_{\mathrm{HF}} 52.3 \mathrm{~Hz},{ }^{3} J_{\mathrm{HF}} 5.9 \mathrm{~Hz}, \mathrm{HCF}_{2}\right), 2.37\left(3 \mathrm{H}, \mathrm{s}, \mathrm{CH}_{3}\right) .{ }^{13} \mathrm{C}$ NMR (100 MHz, DMSO- $\left.d_{6}\right): \delta_{\mathrm{C}} 149.8(\mathrm{C}=\mathrm{O}), 143.1\left(\underline{\mathrm{C}}_{\mathrm{Ar}}-\mathrm{CH}_{3}\right), 142.3(\mathrm{C}-6), 140.2\left(\mathrm{C}_{\mathrm{Ar}}-\mathrm{SO}_{2}\right)$, 129.4, $127.6\left(2 \mathrm{C}_{\mathrm{Ar}} \mathrm{H}\right), 111.5\left(\mathrm{~m}, \mathrm{CF}_{2}\right), 109.8$ (tt, $\left.J_{\mathrm{CF}} 250.5 \mathrm{~Hz},{ }^{2} J_{\mathrm{CF}} 29.0 \mathrm{~Hz}, \mathrm{HCF}_{2}\right), 108.8(\mathrm{C}-5)$, $83.0\left(\mathrm{t},{ }^{2} J_{\mathrm{CF}} 29.6 \mathrm{~Hz}, \mathrm{C}-4\right), 21.1\left(\mathrm{CH}_{3}\right) .{ }^{19} \mathrm{~F}$ NMR $\left(188 \mathrm{MHz}, \mathrm{DMSO}-d_{6}\right): \delta_{\mathrm{F}}-127.68(2 \mathrm{~F}, \mathrm{ABm}$, $\left.J_{\mathrm{AB}} 270.0 \mathrm{~Hz}, \mathrm{CF}_{2}\right),-134.73\left(2 \mathrm{~F}, \mathrm{~d},{ }^{2} J_{\mathrm{FH}} 52.3 \mathrm{~Hz}, \mathrm{HCF}_{2}\right)$. MS APCI, $m / z(\%)=367(\mathrm{M}-\mathrm{H}$, 100). Anal. Calcd for $\mathrm{C}_{13} \mathrm{H}_{12} \mathrm{~F}_{4} \mathrm{~N}_{2} \mathrm{O}_{4} \mathrm{~S}: \mathrm{C}, 42.39 ; \mathrm{H}, 3.28 ; \mathrm{N}, 7.61 ; \mathrm{S}, 8.71 \%$. Found: C, 42.50; H, 3.37 ; N $7.70 ; \mathrm{S}, 8.79 \%$.

$N, N$-Dibenzyl-4-hydroxy-2-oxo-4-(trifluoromethyl)-1,2,3,4-tetrahydropyrimidine-5-sulfonamide (3c). Colorless solid, yield 65\%, $0.29 \mathrm{~g}, \mathrm{mp} 179-181{ }^{\circ} \mathrm{C}$ (from $\left.\mathrm{CH}_{3} \mathrm{CN}\right)$; IR $\left(v_{\max }, \mathrm{cm}^{-1}\right)$ : 3350, 3250, 3120, $2990(\mathrm{NH}, \mathrm{OH}), 1695(\mathrm{C}=\mathrm{O}) .{ }^{1} \mathrm{H}$ NMR (400 MHz, DMSO- $\left.d_{6}\right): \delta_{\mathrm{H}} 10.04$, 8.70, $7.39(3 \mathrm{H}, \mathrm{s}, 2 \mathrm{NH}, \mathrm{OH}), 7.08-7.14\left(10 \mathrm{H}, \mathrm{m}, 2 \mathrm{C}_{6} \mathrm{H}_{5}\right), \delta_{\mathrm{A}} 4.45\left(1 \mathrm{H}, \mathrm{AB}, J_{\mathrm{AB}} 15.8 \mathrm{~Hz}, \mathrm{H}_{\mathrm{A}}\right), \delta_{\mathrm{B}}$ $4.16\left(1 \mathrm{H}, \mathrm{AB}, J_{\mathrm{AB}} 15.8 \mathrm{~Hz}, \mathrm{H}_{\mathrm{B}}\right) .{ }^{13} \mathrm{C}$ NMR (100 MHz, DMSO- $\left.d_{6}\right)$ : $\delta_{\mathrm{C}} 149.5(\mathrm{C}=\mathrm{O}), 141.2(\mathrm{C}-6)$, $128.5,128.8,129.0,135.1\left(2 \cdot 4 \mathrm{C}_{\mathrm{Ph}}\right) .122 .5$ (q, $\left.J_{\mathrm{CF}} 286.6 \mathrm{~Hz}, \mathrm{CF}_{3}\right), 108.5(\mathrm{C}-5), 82.0\left(\mathrm{q},{ }^{2} J_{\mathrm{CF}} 33.7\right.$ $\mathrm{Hz}, \mathrm{C}-4), 50.4\left(2 \mathrm{CH}_{2} \mathrm{~N}\right) .{ }^{19} \mathrm{~F}$ NMR (188 MHz, DMSO- $\left.d_{6}\right): \delta_{\mathrm{F}}-81.64\left(3 \mathrm{~F}, \mathrm{~s}, \mathrm{CF}_{3}\right)$. MS APCI, $m / z$ 
$(\%)=440(\mathrm{M}-\mathrm{H}, 100)$. Anal. Calcd for $\mathrm{C}_{19} \mathrm{H}_{18} \mathrm{~F}_{3} \mathrm{~N}_{3} \mathrm{O}_{4} \mathrm{~S}$ (441.42): C, 51.70; H, 4.11; N, 9.52; S, 7.26\%. Found: C, 51.80; H, 4.30; N, 9.40; S, 7.27\%.

Three-component reaction of 2-oxo-2-polyfluoroalkylethane-1-sulfones (1a,b) and - sulfamide (1c) with 2-aminobenzimidazole and trialkyl orthoformate. Compounds 4a-c. These compounds were synthesized in a similar method as described for the synthesis of compounds 3a-c using 2-aminobenzimidazole in place of urea with the reaction times 4 to $5 \mathrm{~h}$.

3-Tosyl-4-(trifluoromethyl)-4,10-dihydrobenzo[4,5]imidazo[1,2-a]pyrimidin-4-ol (4a). Colorless solid, yield 55\%, $0.23 \mathrm{~g}$, mp 273-275 ${ }^{\circ} \mathrm{C}$ (from $\mathrm{CH}_{3} \mathrm{CN}$ ). ${ }^{1} \mathrm{H}$ NMR (400 MHz, DMSO$\left.d_{6}\right): \delta_{\mathrm{H}} 12.02(1 \mathrm{H}, \mathrm{s}, \mathrm{NH}), 9.23(1 \mathrm{H}, \mathrm{s}, \mathrm{OH}), 8.13(1 \mathrm{H}, \mathrm{s},=\mathrm{CH}), 7.36$ and $7.81(4 \mathrm{H}, \mathrm{dd}, J 7.9 \mathrm{~Hz}$, $\mathrm{C}_{6} \mathrm{H}_{4}$-para $) 7.62\left(1 \mathrm{H}, \mathrm{m}, \mathrm{H}_{\mathrm{Ar}}\right), 7.45\left(1 \mathrm{H}, \mathrm{m}, \mathrm{H}_{\mathrm{Ar}}\right), 7.19\left(1 \mathrm{H}, \mathrm{m}, \mathrm{H}_{\mathrm{Ar}}\right), 7.13\left(1 \mathrm{H}, \mathrm{m}, \mathrm{H}_{\mathrm{Ar}}\right), 2.35$ $\left(3 \mathrm{H}, \mathrm{s}, \mathrm{CH}_{3}\right) .{ }^{13} \mathrm{C}$ NMR $\left(100 \mathrm{MHz}, \mathrm{DMSO}-d_{6}\right): \delta_{\mathrm{C}} 145.1\left(\mathrm{C}_{\mathrm{Ar}}\right), 143.1(=\mathrm{CH}), 143.0\left(\underline{\mathrm{C}}_{\mathrm{Ar}}-\mathrm{CH}_{3}\right)$, $141.0(\mathrm{C}=\mathrm{N}), 140.6\left(\underline{\mathrm{C}}_{\mathrm{Ar}}-\mathrm{SO}_{2}\right), 131.0\left(\mathrm{C}_{\mathrm{Ar}}\right), 129.5,127.6,123.0,127.5,117.1,114.3\left(6 \mathrm{C}_{\mathrm{Ar}}-\mathrm{H}\right)$, $123.5\left(\mathrm{q}, J_{\mathrm{CF}} 293.3 \mathrm{~Hz}, \mathrm{CF}_{3}\right), 106.1(\mathrm{C}-2), 85.2\left(\mathrm{q},{ }^{2} J_{\mathrm{CF}} 35.2 \mathrm{~Hz}, \mathrm{C}-4\right), 21.1\left(\mathrm{CH}_{3}\right) .{ }^{19} \mathrm{~F}$ NMR (188 MHz, DMSO- $\left.d_{6}\right): \delta_{\mathrm{F}}-79.34\left(3 \mathrm{~F}, \mathrm{~s}, \mathrm{CF}_{3}\right)$. MS APCI, $m / z(\%)=410(\mathrm{MH}, 100)$. Anal. Calcd for $\mathrm{C}_{18} \mathrm{H}_{14} \mathrm{~F}_{3} \mathrm{~N}_{3} \mathrm{O}_{3} \mathrm{~S}$ (409.38): C, 52.81; H, 3.45; N, 10.26; S, 7.83\%. Found: C, 53.01; H, 3.65; N, $10.20 ; \mathrm{S}, 7.69 \%$.

\section{4-(1,1,2,2-Tetrafluoroethyl)-3-tosyl-4,10-dihydrobenzo[4,5]imidazo[1,2-a]pyrimidin-4-ol} (4b). Colorless solid, yield 47\%, $0.21 \mathrm{~g}$, mp 215-217 ${ }^{\circ} \mathrm{C}$ (from $\mathrm{CH}_{3} \mathrm{CN}$ ). ${ }^{1} \mathrm{H}$ NMR $(400 \mathrm{MHz}$, DMSO- $\left.d_{6}\right): \delta_{\mathrm{H}} 13.10(1 \mathrm{H}, \mathrm{s}, \mathrm{NH}), 8.69(1 \mathrm{H}, \mathrm{s}, \mathrm{OH}), 7.98(1 \mathrm{H}, \mathrm{s},=\mathrm{CH}), 7.36$ and $7.79(4 \mathrm{H}, \mathrm{dd}, J$ $7.9 \mathrm{~Hz}, \mathrm{C}_{6} \mathrm{H}_{4}-$ para $), 7.61\left(1 \mathrm{H}, \mathrm{m}, \mathrm{H}_{\mathrm{Ar}}\right), 7.42\left(1 \mathrm{H}, \mathrm{m}, \mathrm{H}_{\mathrm{Ar}}\right), 7.17\left(1 \mathrm{H}, \mathrm{m}, \mathrm{H}_{\mathrm{Ar}}\right), 7.11\left(1 \mathrm{H}, \mathrm{m}, \mathrm{H}_{\mathrm{Ar}}\right)$, $6.82\left(1 \mathrm{H}, \mathrm{tdd},{ }^{2} J_{\mathrm{HF}} 52.3 \mathrm{~Hz},{ }^{3} J_{\mathrm{HF}} 8.2 \mathrm{~Hz},{ }^{3} J_{\mathrm{HF}} 3.4 \mathrm{~Hz}, \mathrm{HCF}_{2}\right), 2.36\left(3 \mathrm{H}, \mathrm{s}, \mathrm{CH}_{3}\right) .{ }^{13} \mathrm{C} \mathrm{NMR}(100$ $\left.\mathrm{MHz}, \mathrm{DMSO}-d_{6}\right): \delta_{\mathrm{C}} 145.1\left(\mathrm{C}_{\mathrm{Ar}}\right), 142.8\left(\underline{\mathrm{C}}_{\mathrm{Ar}}-\mathrm{CH}_{3}\right), 142.4(=\mathrm{CH}), 140.5\left(\underline{\mathrm{C}}_{\mathrm{Ar}}-\mathrm{SO}_{2}\right), 140.1(\mathrm{C}=\mathrm{N})$, $131.5\left(\mathrm{C}_{\mathrm{Ar}}\right), 129.2,127.5,122.5,121.0,116.3,114.2\left(6 \mathrm{C}_{\mathrm{Ar}}-\mathrm{H}\right), 111-118\left(\mathrm{~m}, \mathrm{CF}_{2}\right), 104-110(\mathrm{~m}$, $\left.\mathrm{HCF}_{2}\right), 106.9(\mathrm{C}-2), 86.6\left(\mathrm{dd},{ }^{2} J_{\mathrm{CF}} 31.6 \mathrm{~Hz},{ }^{2} J_{\mathrm{CF}} 25.0 \mathrm{~Hz}, \mathrm{C}-4\right), 20.7\left(\mathrm{~s}, \mathrm{CH}_{3}\right) .{ }^{19} \mathrm{~F}$ NMR $(188$ $\left.\mathrm{MHz}, \mathrm{DMSO}-d_{6}\right): \delta_{\mathrm{F}} \delta_{\mathrm{A}}-118.42\left(1 \mathrm{~F}, \mathrm{ABm}, J_{\mathrm{AB}} 260.0 \mathrm{~Hz}, \mathrm{CF}_{\mathrm{A}} \mathrm{F}_{\mathrm{B}}\right), \delta_{\mathrm{B}}-122.57\left(1 \mathrm{~F}, \mathrm{ABm}, J_{\mathrm{AB}}\right.$ $\left.260.0 \mathrm{~Hz}, \mathrm{CF}_{\mathrm{A}} \underline{\mathrm{F}}_{\mathrm{B}}\right), \delta_{\mathrm{A}}-133.59\left(1 \mathrm{~F}, \mathrm{ABd}, J_{\mathrm{AB}} 295.4 \mathrm{~Hz},{ }^{2} J_{\mathrm{FH}} 52.3 \mathrm{~Hz}, \mathrm{HCF}_{\mathrm{A}} \mathrm{F}_{\mathrm{B}}\right), \delta_{\mathrm{B}}-136.40(1 \mathrm{~F}$, $\left.\mathrm{ABd}, J_{\mathrm{AB}} 295.4 \mathrm{~Hz},{ }^{2} J_{\mathrm{FH}} 52.3 \mathrm{~Hz}, \mathrm{HCF}_{\mathrm{A}} \underline{\mathrm{F}}_{\mathrm{B}}\right)$. MS APCI, $m / z(\%)=442(\mathrm{MH}, 100)$. Anal. Calcd for $\mathrm{C}_{19} \mathrm{H}_{15} \mathrm{~F}_{4} \mathrm{~N}_{3} \mathrm{O}_{3} \mathrm{~S}$ (441.40): C, 51.70; H, 3.43; N, 9.52; S, 7.26\%. Found: C, 52.01; H, 3.35; N, 9.40; S, 7.39\%.

$N, N$-Diethyl-4-hydroxy-4-(trifluoromethyl)-4,10-dihydrobenzo[4,5]imidazo[1,2-a]pyrimidine-3-sulfonamide (4c). Colorless solid, yield 45\%, $0.18 \mathrm{~g}, \mathrm{mp} 205-207{ }^{\circ} \mathrm{C}$ (from $\mathrm{CH}_{3} \mathrm{CN}$ ). ${ }^{1} \mathrm{H}$ NMR (400 MHz, DMSO- $\left.d_{6}\right): \delta_{\mathrm{H}} 11.65(1 \mathrm{H}, \mathrm{s}, \mathrm{NH}), 9.24(1 \mathrm{H}, \mathrm{s}, \mathrm{OH}), 7.72\left(1 \mathrm{H}, \mathrm{m}, \mathrm{H}_{\mathrm{Ar}}\right), 7.71$ $(1 \mathrm{H}, \mathrm{s},=\mathrm{CH}), 7.46\left(1 \mathrm{H}, \mathrm{m}, \mathrm{H}_{\mathrm{Ar}}\right), 7.21\left(1 \mathrm{H}, \mathrm{m}, \mathrm{H}_{\mathrm{Ar}}\right), 7.15\left(1 \mathrm{H}, \mathrm{m}, \mathrm{H}_{\mathrm{Ar}}\right), 3.08-3.31\left(4 \mathrm{H}, \mathrm{m}, 2 \mathrm{CH}_{2}\right)$, $1.15\left(6 \mathrm{H}, \mathrm{t}, J 7.0 \mathrm{~Hz}, 2 \mathrm{CH}_{3}\right) .{ }^{13} \mathrm{C} \mathrm{NMR}\left(100 \mathrm{MHz}, \mathrm{DMSO}-d_{6}\right): \delta_{\mathrm{C}} 145.1\left(\mathrm{C}_{\mathrm{Ar}}\right), 141.7(\mathrm{C}=\mathrm{N})$, $139.9(=\mathrm{CH}), 131.4\left(\mathrm{C}_{\mathrm{Ar}}\right), 123.7\left(\mathrm{q}, J_{\mathrm{CF}} 294.0 \mathrm{~Hz}, \mathrm{CF}_{3}\right), 122.9,121.2,117.2,114.3\left(6 \mathrm{C}_{\mathrm{Ar}}-\mathrm{H}\right)$, $105.3(\mathrm{C}-2), 85.1\left(\mathrm{q},{ }^{2} J_{\mathrm{CF}} 35.1 \mathrm{~Hz}, \mathrm{C}-4\right), 43.1\left(2 \mathrm{CH}_{2}\right), 15.9\left(2 \mathrm{CH}_{3}\right) .{ }^{19} \mathrm{~F}$ NMR $(188 \mathrm{MHz}, \mathrm{DMSO}-$ $\left.d_{6}\right): \delta_{\mathrm{F}}-78.41\left(3 \mathrm{~F}, \mathrm{~s}, \mathrm{CF}_{3}\right)$. MS APCI, $m / z(\%)=391(\mathrm{MH}, 100)$. Anal. Calcd for $\mathrm{C}_{15} \mathrm{H}_{17} \mathrm{~F}_{3} \mathrm{~N}_{4} \mathrm{O}_{3} \mathrm{~S}$ (390.38): C, 46.15; H, 4.39; N, 14.35; S, 8.21\%. Found: C, 46.20; H, 4.45; N, 14.50; S, 8.30\%. 
General synthetic procedure for the synthesis of 4-alkoxy-3,4-dihydropyrimidin-2(1H)-ones (6a-c). A suspension of dihydropyrimidinone $3 \mathbf{a}(168 \mathrm{mg}, 0.5 \mathrm{mmol})$ and phosphorus pentoxide $(213 \mathrm{mg}, 1.5 \mathrm{mmol})$ in acetonitrile $(10 \mathrm{~mL})$ was refluxed under vigorous stirring for $30 \mathrm{~min}$ and filtered off while hot to discard some solid material. The corresponding alcohol $(0.5 \mathrm{mmol})$ was added to the filtrate at ambient temperature, the solvent was removed under reduced pressure and the residue was crystallized from acetonitrile.

4-Methoxy-5-tosyl-4-(trifluoromethyl)-3,4-dihydropyrimidin-2(1H)-one (6a). Colorless solid, yield 92\%, $0.16 \mathrm{~g}, \mathrm{mp} 207-209{ }^{\circ} \mathrm{C}$ (from $\mathrm{CH}_{3} \mathrm{CN}$ ); IR $\left(v_{\max }, \mathrm{cm}^{-1}\right)$ : 3360, 3240, 3125, $2995(\mathrm{NH}, \mathrm{OH}), 1690(\mathrm{C}=\mathrm{O}) .{ }^{1} \mathrm{H}$ NMR $\left(400 \mathrm{MHz}, \mathrm{DMSO}-d_{6}\right): \delta_{\mathrm{H}} 10.61(1 \mathrm{H}, \mathrm{d}, J 5.6 \mathrm{~Hz}, \mathrm{NH}-1)$, $8.81(1 \mathrm{H}, \mathrm{s}, \mathrm{NH}-3), 7.94(1 \mathrm{H}, \mathrm{d}, J 5.6 \mathrm{~Hz}, \mathrm{H}-6), 7.39$ and $7.72\left(4 \mathrm{H}, \mathrm{dd}, J 8.1 \mathrm{~Hz}, \mathrm{C}_{6} \mathrm{H}_{4}\right.$-para $)$, $2.79\left(3 \mathrm{H}, \mathrm{s}, \mathrm{OCH}_{3}\right), 2.39\left(3 \mathrm{H}, \mathrm{s}, \mathrm{CH}_{3}\right) .{ }^{13} \mathrm{C} \mathrm{NMR}\left(100 \mathrm{MHz}, \mathrm{DMSO}-d_{6}\right): \delta_{\mathrm{C}} 149.8(\mathrm{C}=\mathrm{O}), 145.6$ (C-6), $143.6\left(\underline{\mathrm{C}}_{\mathrm{Ar}}-\mathrm{CH}_{3}\right), 138.9\left(\mathrm{C}_{\mathrm{Ar}}-\mathrm{SO}_{2}\right), 129.4,127.6\left(2 \mathrm{C}_{\mathrm{Ar}}-\mathrm{H}\right), 118.9$ (q, $\left.J_{\mathrm{CF}} 288.8 \mathrm{~Hz}, \mathrm{CF}_{3}\right)$, $103.3(\mathrm{C}-5), 87.1$ (q, $\left.{ }^{2} J_{\mathrm{CF}} 33.0 \mathrm{~Hz}, \mathrm{C}-4\right), 49.3\left(\mathrm{OCH}_{3}\right), 21.1\left(\mathrm{CH}_{3}\right) .{ }^{19} \mathrm{~F}$ NMR (188 MHz, DMSO$\left.d_{6}\right): \delta_{\mathrm{F}}-79.34\left(3 \mathrm{~F}, \mathrm{~s}, \mathrm{CF}_{3}\right)$. MS APCI, $m / z(\%)=349(\mathrm{M}-\mathrm{H}, 100)$. Anal. Calcd for $\mathrm{C}_{13} \mathrm{H}_{13} \mathrm{~F}_{3} \mathrm{~N}_{2} \mathrm{O}_{4} \mathrm{~S}$ (350.31): C, 44.57; H, 3.74; N, 8.00; S, 9.15\%. Found: C, 44.80; H, 3.67; N, $8.20 ; \mathrm{S}, 9.33 \%$.

4-(1-Phenylethoxy)-5-tosyl-4-(trifluoromethyl)-3,4-dihydropyrimidin-2(1H)-one

(6b).

Colorless solid, yield $86 \%, 0.19 \mathrm{~g}, \mathrm{mp} 226-228{ }^{\circ} \mathrm{C}$ (from $\mathrm{CH}_{3} \mathrm{CN}$ ); IR $\left(v_{\max }, \mathrm{cm}^{-1}\right)$ : 3350, 3230, 3070, 2920, 1695 (C=O). ${ }^{1} \mathrm{H}$ NMR (400 MHz, DMSO- $\left.d_{6}\right): \delta_{\mathrm{H}} 10.39(1 \mathrm{H}, \mathrm{d}, J 5.4 \mathrm{~Hz}, \mathrm{NH}-1)$, $8.18(1 \mathrm{H}, \mathrm{s}, \mathrm{NH}-3), 7.96(1 \mathrm{H}, \mathrm{d}, J 5.4 \mathrm{~Hz}, \mathrm{H}-6), 7.39$ and $7.75\left(4 \mathrm{H}, \mathrm{dd}, J 8.1 \mathrm{~Hz}, \mathrm{C}_{6} \mathrm{H}_{4}\right.$-para), 7.22-7.30 (5H, m, $\left.\mathrm{C}_{6} \mathrm{H}_{5}\right), 4.80(1 \mathrm{H}, \mathrm{q}, J 4.5 \mathrm{~Hz}, \mathrm{CH}), 2.39\left(3 \mathrm{H}, \mathrm{s}, \mathrm{CH}_{3}\right), 1.25(3 \mathrm{H}, \mathrm{d}, J 4.5 \mathrm{~Hz}$, $\left.\mathrm{CH}_{3}\right) .{ }^{13} \mathrm{C}$ NMR (100 MHz, DMSO- $\left.d_{6}\right): \delta_{\mathrm{C}} 148.3(\mathrm{C}=\mathrm{O}), 145.3(\mathrm{C}-6), 143.3\left(\underline{\mathrm{C}}_{\mathrm{Ar}}-\mathrm{CH}_{3}\right), 143.1$ ( $\mathrm{C}_{\mathrm{Ph}}$-ipso $), 139.1\left(\mathrm{C}_{\mathrm{Ar}}-\mathrm{SO}_{2}\right), 129.1,128.0,127.1,126.9,125.6\left(5 \mathrm{C}_{\mathrm{Ar}}-\mathrm{H}\right), 121.2\left(\mathrm{q}, J_{\mathrm{CF}} 292.0 \mathrm{~Hz}\right.$, $\left.\mathrm{CF}_{3}\right), 104.2(\mathrm{C}-5), 86.5$ (q, $\left.{ }^{2} J_{\mathrm{CF}} 33.7 \mathrm{~Hz}, \mathrm{C}-4\right), 72.1(\mathrm{CHO}), 23.9\left(\mathrm{CH}_{3}\right), 20.7\left(\mathrm{CH}_{3}\right) .{ }^{19} \mathrm{~F}$ NMR $\left(100 \mathrm{MHz}, \mathrm{DMSO}-d_{6}\right): \delta_{\mathrm{F}}-77.97\left(3 \mathrm{~F}, \mathrm{~s}, \mathrm{CF}_{3}\right)$. MS APCI, $m / z(\%)=439(\mathrm{M}-\mathrm{H}, 100)$. Anal. Calcd for $\mathrm{C}_{20} \mathrm{H}_{19} \mathrm{~F}_{3} \mathrm{~N}_{2} \mathrm{O}_{4} \mathrm{~S}$ (440.44): C, 54.54; H, 4.35; N, 6.36; S, 7.28\%. Found: C, 54.40; H, $4.32 ; \mathrm{N}, 6.40 ; \mathrm{S}, 7.43 \%$.

(2R)-Ethyl 2-((2-oxo-5-tosyl-4-(trifluoromethyl)-1,2,3,4-tetrahydropyrimidin-4-yl)oxy)propanoate (6c). Colorless solid, yield $82 \%, 0.18 \mathrm{~g}$, mp 191-192 ${ }^{\circ} \mathrm{C}$ (from $\left.\mathrm{CH}_{3} \mathrm{CN}\right)$; IR $\left(v_{\max }\right.$, $\left.\mathrm{cm}^{-1}\right)$ : 3360, 3240, 3080, 2900, $1725(\mathrm{C}=\mathrm{O}), 1700(\mathrm{C}=\mathrm{O}) .{ }^{1} \mathrm{H}$ NMR (400 MHz, DMSO- $\left.d_{6}\right): \delta_{\mathrm{H}}$ $10.82(1 \mathrm{H}, \mathrm{d}, J 6.0 \mathrm{~Hz}, \mathrm{NH}-1), 8.52(1 \mathrm{H}, \mathrm{s}, \mathrm{NH}-3), 8.07$ (1H, d, J 6.0 Hz, H-6), 7.38 and 7.74 $\left(4 \mathrm{H}, \mathrm{dd}, J 8.1 \mathrm{~Hz}, \mathrm{C}_{6} \mathrm{H}_{4}-\right.$ para $), 3.97-4.08\left(3 \mathrm{H}, \mathrm{m}, \mathrm{CH}+\mathrm{CH}_{2}\right), 2.38\left(3 \mathrm{H}, \mathrm{s}, \mathrm{CH}_{3}\right), 1.19(6 \mathrm{H}, \mathrm{t}, J$ $\left.4.5 \mathrm{~Hz}, 2 \mathrm{CH}_{3}\right), 1.12\left(3 \mathrm{H}, \mathrm{d}, J 6.6 \mathrm{~Hz}, \mathrm{CH}_{3}\right) .{ }^{13} \mathrm{C} \mathrm{NMR}\left(100 \mathrm{MHz}\right.$, DMSO- $\left.d_{6}\right): \delta_{\mathrm{C}} 172.1(\mathrm{C}=\mathrm{O})$, $148.7(\mathrm{C}=\mathrm{O}), 147.0(\mathrm{C}-6), 143.6\left(\underline{\mathrm{C}}_{\mathrm{Ar}}-\mathrm{CH}_{3}\right), 138.8\left(\mathrm{C}_{\mathrm{Ar}}-\mathrm{SO}_{2}\right), 129.4,127.8\left(2 \mathrm{C}_{\mathrm{Ar}}-\mathrm{H}\right), 121.1(\mathrm{q}$, $\left.J_{\mathrm{CF}} 290.0 \mathrm{~Hz}, \mathrm{CF}_{3}\right), 102.0(\mathrm{C}-5), 87.2\left(\mathrm{q},{ }^{2} J_{\mathrm{CF}} 34.6 \mathrm{~Hz}, \mathrm{C}-4\right), 68.5\left(\mathrm{CH}_{2}\right), 61.0(\mathrm{CHO}), 49.3$ $\left(\mathrm{OCH}_{3}\right), 21.1\left(\mathrm{CH}_{3}\right), 17.6\left(\mathrm{CH}_{3}\right), 13.8\left(\mathrm{CH}_{3}\right) .{ }^{19} \mathrm{~F}$ NMR $\left(100 \mathrm{MHz}, \mathrm{DMSO}-d_{6}\right): \delta_{\mathrm{F}}-78.06(3 \mathrm{~F}, \mathrm{~s}$, $\left.\mathrm{CF}_{3}\right)$. MS APCI, $m / z(\%)=435(\mathrm{M}-\mathrm{H}, 100)$. Anal. Calcd for $\mathrm{C}_{17} \mathrm{H}_{19} \mathrm{~F}_{3} \mathrm{~N}_{2} \mathrm{O}_{6} \mathrm{~S}$ (436.40): $\mathrm{C}$, 46.79; H, 4.39; N, 6.42; S, 7.35\%. Found: C, 46.61; H, 4.23; N, 6.60; S, 7.40\%.

Reaction of compound 3a with potassium hydroxide. Preparation of potassium 5-tosyl-4(trifluoromethyl)pyrimidin-2-olate (7). A mixture of dihydropyrimidinone 3a (168 mg, 0.5 
mmol) and potassium hydroxide $(56 \mathrm{mg}, 1.0 \mathrm{mmol})$ in methanol $(5 \mathrm{~mL})$ was stirred for $1 \mathrm{~h}$. The solvent was evaporated in vacuum and the resulting white solid was dried.

Potassium 5-tosyl-4-(trifluoromethyl)pyrimidin-2-olate (7). Colorless solid, yield 97\%, 0.18 g, mp>250 ${ }^{\circ} \mathrm{C} .{ }^{1} \mathrm{H}$ NMR $\left(300 \mathrm{MHz}, \mathrm{DMSO}-d_{6}\right): \delta_{\mathrm{H}} 8.71(1 \mathrm{H}, \mathrm{s}, \mathrm{H}-6), 7.35$ and $7.64(4 \mathrm{H}, \mathrm{dd}, J$ $8.2 \mathrm{~Hz}, \mathrm{C}_{6} \mathrm{H}_{4}$-para), 2.35 (3H, s, $\left.\mathrm{CH}_{3}\right) .{ }^{13} \mathrm{C}$ NMR (100 MHz, DMSO- $\left.d_{6}\right): \delta_{\mathrm{C}} 165.0(\mathrm{C}-3), 162.7$ (C-6), 153.1 (q, $\left.{ }^{2} J_{\mathrm{CF}} 34.2 \mathrm{~Hz}, \mathrm{C}-4\right), 142.9\left(\underline{\mathrm{C}}_{\mathrm{Ar}}-\mathrm{CH}_{3}\right), 140.9\left(\mathrm{C}_{\mathrm{Ar}}-\mathrm{SO}_{2}\right), 129.6,126.3\left(2 \mathrm{C}_{\mathrm{Ar}}-\mathrm{H}\right)$, $119.8\left(\mathrm{q}, J_{\mathrm{CF}} 275.5 \mathrm{~Hz}, \mathrm{CF}_{3}\right), 111.5(\mathrm{C}-5), 21.0\left(\mathrm{CH}_{3}\right) .{ }^{19} \mathrm{~F}$ NMR $\left(188 \mathrm{MHz}, \mathrm{DMSO}-d_{6}\right): \delta_{\mathrm{F}}-$ $64.93\left(3 \mathrm{~F}, \mathrm{~s}, \mathrm{CF}_{3}\right)$. MS APCI, $m / z(\%)=318(\mathrm{MH}-\mathrm{K}, 100)$. Anal. Calcd for $\mathrm{C}_{12} \mathrm{H}_{8} \mathrm{~F}_{3} \mathrm{KN}_{2} \mathrm{O}_{3} \mathrm{~S}$ (356.36): C, 40.44; H, 2.26; N, 7.86; S, 9.00\%. Found: C, 40.61; H, 2.23; N, 7.61; S, 8.93\%.

Reaction of compound 2a with potassium hydroxide. Preparation of 1-(1-phenyl-2tosylethyl)urea (8). To a solution of tetrahydropyrimidinone $\mathbf{2 a}(207 \mathrm{mg}, 0.5 \mathrm{mmol})$ in methanol $(5 \mathrm{ml})$ a solution of potassium hydroxide $(28 \mathrm{mg}, 0.5 \mathrm{mmol})$ in methanol $(1 \mathrm{~mL})$ was added. The mixture was heated at $60{ }^{\circ} \mathrm{C}$ for $5 \mathrm{~h}$, the solvent was then evaporated in vacuum and the residue was crystallized.

1-(1-Phenyl-2-tosylethyl)urea (8). Colorless needles, yield 70\%, 0.16 g, mp 131-133 ${ }^{\circ} \mathrm{C}$ (from $\mathrm{CH}_{3} \mathrm{CN}-\mathrm{H}_{2} \mathrm{O}$ 1:1); IR $\left(v_{\max }, \mathrm{cm}^{-1}\right): 3515,3410,3055,2930,1695(\mathrm{C}=\mathrm{O}) .{ }^{1} \mathrm{H}$ NMR $(300 \mathrm{MHz}$, DMSO- $\left.d_{6}\right): \delta_{\mathrm{H}} 7.36$ and $7.68\left(4 \mathrm{H}, \mathrm{dd}, J 8.2 \mathrm{~Hz}, \mathrm{C}_{6} \mathrm{H}_{4}\right.$-para $), 7.17-7.26\left(5 \mathrm{H}, \mathrm{m}, \mathrm{C}_{6} \mathrm{H}_{5}\right), 6.51(1 \mathrm{H}$, d, J $8.4 \mathrm{~Hz}, \mathrm{NH}), 5.56\left(2 \mathrm{H}, \mathrm{s}, \mathrm{NH}_{2}\right), 5.04(1 \mathrm{H}, \mathrm{ddd}, J 8.4 \mathrm{~Hz}, J 8.3 \mathrm{~Hz}, J 5.3 \mathrm{~Hz}, \mathrm{CH}), 3.77(1 \mathrm{H}$, $\left.\mathrm{ABd}, J_{\mathrm{AB}} 14.6 \mathrm{~Hz}, J 8.3 \mathrm{~Hz}, \mathrm{H}_{\mathrm{A}}\right), 3.67\left(1 \mathrm{H}, \mathrm{ABd}, J_{\mathrm{AB}} 14.6 \mathrm{~Hz}, J 5.3 \mathrm{~Hz}, \mathrm{H}_{\mathrm{B}}\right), 2.38\left(3 \mathrm{H}, \mathrm{s}, \mathrm{CH}_{3}\right)$. ${ }^{13} \mathrm{C}$ NMR $\left(100 \mathrm{MHz}, \mathrm{DMSO}-d_{6}\right): \delta_{\mathrm{C}} 157.5(\mathrm{C}=\mathrm{O}), 144.1\left(\underline{\mathrm{C}}_{\mathrm{Ar}}-\mathrm{CH}_{3}\right), 141.6\left(\mathrm{C}_{\mathrm{Ph}}-i p s o\right), 136.7$ $\left(\mathrm{C}_{\mathrm{Ar}}-\mathrm{SO}_{2}\right), 129.7,128.3,127.8,127.2,126.7\left(5 \mathrm{C}_{\mathrm{Ar}}-\mathrm{H}\right), 66.2\left(\mathrm{CH}_{2}\right), 49.0(\mathrm{CH}), 21.1\left(\mathrm{CH}_{3}\right) . \mathrm{MS}$ APCI, $m / z(\%)=319(\mathrm{MH}, 100)$. Anal. Calcd for $\mathrm{C}_{16} \mathrm{H}_{18} \mathrm{~N}_{2} \mathrm{O}_{3} \mathrm{~S}$ (318.39): C, 60.36; H, 5.70; N, 8.80; S, 10.07\%. Found: C, 60.50; H, 5.59; N, 8.82; S, 10.00\%.

Reaction of compound $2 \mathrm{a}$ with phosphorus pentachloride. Preparation of 1,1,1-trifluoro-4phenyl-3-tosylbut-3-en-2-one (10). A suspension of tetrahydropyrimidinone 2a (207 $\mathrm{mg}, 0.5$ mmol) and phosphorus pentachloride $(209 \mathrm{mg}, 1 \mathrm{mmol})$ in $5 \mathrm{~mL}$ of benzene was refluxed for 3 $\mathrm{h}$. The solvent was evaporated in vacuum, the residue was treated with hexane and decanted several times from the solid product with hexane $(6 \mathrm{~mL})$ to give the white powder-like solid, which was filtered and dried.

1,1,1-Trifluoro-4-phenyl-3-tosylbut-3-en-2-one (10). White powder, yield 75\%, $0.27 \mathrm{~g}, \mathrm{mp}$ 110-112 ${ }^{\circ} \mathrm{C} .{ }^{1} \mathrm{H}$ NMR $\left(300 \mathrm{MHz}\right.$, acetone- $\left.d_{6}\right): \delta_{\mathrm{H}} 8,43(1 \mathrm{H}, \mathrm{s},=\mathrm{CH}), 7.39$ and $7.86(4 \mathrm{H}, \mathrm{dd}, J$ $8.2 \mathrm{~Hz}, \mathrm{C}_{6} \mathrm{H}_{4}$-para), 7.36-7.63 (5H, m, $\left.\mathrm{C}_{6} \mathrm{H}_{5}\right), 2.46\left(3 \mathrm{H}, \mathrm{s}, \mathrm{CH}_{3}\right) .{ }^{13} \mathrm{C}$ NMR (100 MHz, acetone$\left.d_{6}\right): \delta_{\mathrm{C}} 185.4\left(\mathrm{q},{ }^{2} J_{\mathrm{CF}} 38.5 \mathrm{~Hz}, \mathrm{C}=\mathrm{O}\right), 148.3(=\mathrm{CH}), 146.7\left(\underline{\mathrm{C}}_{\mathrm{Ar}}-\mathrm{CH}_{3}\right), 137.7\left(\mathrm{C}_{\mathrm{Ph}}-i p s o\right), 136.6$ $\left(\mathrm{C}_{\mathrm{Ar}}-\mathrm{SO}_{2}\right), 132.2(=\mathrm{C}), 133.4,131.1,130.8,130.4,129.4\left(5 \mathrm{C}_{\mathrm{Ar}}-\mathrm{H}\right), 115.6\left(\mathrm{q}, J_{\mathrm{CF}} 289.6 \mathrm{~Hz}, \mathrm{CF}_{3}\right)$, $21.6\left(\mathrm{CH}_{3}\right) .{ }^{19} \mathrm{~F}$ NMR $\left(188 \mathrm{MHz}\right.$, acetone- $\left.d_{6}\right): \delta_{\mathrm{F}}-73.88\left(3 \mathrm{~F}, \mathrm{~s}, \mathrm{CF}_{3}\right) . \mathrm{GC} / \mathrm{MS}, m / z(\%)=354$ (M $\left.\mathrm{M}^{+}, 3\right), 290$ (45), 199 (25), 155 (10), 139 (15), 91 (100), 65 (40). Anal. Calcd for $\mathrm{C}_{17} \mathrm{H}_{13} \mathrm{~F}_{3} \mathrm{O}_{3} \mathrm{~S}$ (354.34): C, 57.62; H, 3.70; S, 9.05\%. Found: C, 57.90; H, 3.59; S, 9.00\%. 
X-ray crystallographic data. Single crystal X-ray diffraction data were collected at $-150{ }^{\circ} \mathrm{C}$ on a Bruker Apex II CCD diffractometer. Data were corrected for Lorentz and polarisation effects and an absorption correction using the Sadabs procedure ${ }^{30}$ was applied. The structure was solved by direct methods and refined by full-matrix least-squares technique in the anisotropic approximation using the CRYSTALS program package. ${ }^{31}$ About $50 \%$ of the hydrogen atoms were located in the difference Fourier maps, the remained $\mathrm{H}$ atoms were placed in the calculated positions. All hydrogen atoms were included in the refinement with the fixed positional and thermal parameters. Convergence was obtained at $\mathrm{R}=0.039$ and $\mathrm{R}_{\mathrm{w}}=0.037, \mathrm{GOF}=1.150(181$ refined parameters; obs./variabl. 9.5). Chebushev weighting scheme ${ }^{32}$ with parameters 1.26 , $1.56,0.56,-0.47$, and -0.24 was used. Crystallographic data were deposited with the Cambridge Crystallographic Data Centre as supplementary publication no. CCDC-749282. The data can be obtained free of charge on application to CCDC, 12 Union Road, Cambridge CB2 1EZ, UK (fax: +44 (0)1223 336033 or email: deposit@ ccdc.cam.ac.uk).

Crystal data for 4-hydroxy-6-phenyl-5-[(4-methylphenyl)sulfonyl]-4-(trifluoromethyl)tetrahydropyrimidin-2(1H)-one (2a). $\mathrm{C}_{18} \mathrm{H}_{17} \mathrm{~F}_{3} \mathrm{~N}_{2} \mathrm{O}_{4} \mathrm{~S}, \mathrm{M}=414.4$, monoclinic, $a=11.4870(18)$, $b=6.9426(12), c=23.132(4) \AA, \beta=95.124(8)^{\circ}, \mathrm{V}=1837.4(5) \AA^{3}, \mathrm{Z}=4, \mathrm{~d}=1.498 \mathrm{~g} \cdot \mathrm{cm}^{-1}$, space group $\mathrm{P} 2{ }_{1} / \mathrm{c}(\mathrm{N} 14), \mathrm{F}(000)=856$, crystal size ca. $0.45 \times 0.18 \times 0.06 \mathrm{~mm}, 13950$ reflections $(2378$ unique), $\mathrm{R}=0.09$ and $\mathrm{R}_{\mathrm{w}}=0.235, \mathrm{GOF}=1.053$ (267 refined parameters; obs./variabl. 8.9).

\section{Acknowledgements}

Authors thank Dr. V. V. Trachevsky, the Joint Use Center of NMR Spectroscopy at the G. V. Kurdyumov Institute for Metal Physics of NAS of Ukraine for recording the NMR spectra.

\section{References}

1. Zhu, J.; Bienaymé, H. Multicomponent reactions; Wiley-VCH: Weinheim, 2005.

2. Simon, C.; Constantieux, T.; Rodriguez, J. Eur. J. Org. Chem. 2004, 4957. http://dx.doi.org/10.1002/ejoc.200400511

3. Kappe, C. O. Eur. J. Med. Chem. 2000, 35, 1043. http://dx.doi.org/10.1016/S0223-5234(00)01189-2

4. Singh, K.; Arora, D.; Singh, K.; Singh, S. Mini-Rev. Med. Chem. 2009, 9, 95.

5. Kumar, B. R. P.; Sankar, G.; Baig, R. B.; Chandrashekaran, S. Eur. J. Med. Chem. 2009, 44, 4192.

http://dx.doi.org/10.1016/j.ejmech.2009.05.014

6. Bégué, J.-P.; Bonnet-Delpon, D. J. Fluorine Chem. 2006, 127, 992. http://dx.doi.org/10.1016/j.jfluchem.2006.05.006; 
7. Isanbor, C.; O'Hagan, D. J. Fluorine Chem. 2006, 12, 303.

http://dx.doi.org/10.1016/j.jfluchem.2006.01.011

8. Kirk, K. L. J. Fluorine Chem. 2006, 127, 1013. http://dx.doi.org/10.1016/j.jfluchem.2006.06.007

9. Purser, S; Moore, P. R.; Swallow, S; Gouverneur, V. Chem. Soc. Rev. 2008, 37, 320. http://dx.doi.org/10.1039/b610213c

10. Kappe, C. O. Acc. Chem. Res. 2000, 33, 879. http://dx.doi.org/10.1021/ar000048h

11. Wan, J.-P.; Liu, Y. Synthesis 2010, 3943. http://dx.doi.org/10.1055/s-0030-1258290

12. Vdovina, S. V.; Mamedov, V. A. Russ. Chem. Rev. 2008, 77, 1017. http://dx.doi.org/10.1070/RC2008v077n12ABEH003894

13. Gladkov, E. S.; Chebanov, V. A.; Desenko, S. M.; Shishkin, O. V.; Shishkina, S. V.; Dallinger, D.; Kappe, C. O. Heterocycles 2007, 73(1), 469. http://dx.doi.org/10.3987/COM-07-S(U)19

14. Xing, C; Zhu, S. J. Org. Chem. 2004, 37, 6486. http://dx.doi.org/10.1021/jo049317y

15. Abelman, M. M.; Smith, S. C.; James, D. R. Tetrahedron Lett. 2003, 44, 4559. http://dx.doi.org/10.1016/S0040-4039(03)00985-7

16. Timoshenko, V. M.; Markitanov, Yu. M.; Shermolovich, Yu. G. Tetrahedron Lett. 2011, 52, 6619. http://dx.doi.org/10.1016/j.tetlet.2011.09.143

17. Kappe, C. O.; Falsone, S. F; Fabian, W. M. F.; Belaj, F. Heterocycles 1999, 51, 77. http://dx.doi.org/10.3987/COM-98-8336

18. Saloutin, V. I.; Burgart, Ya. V.; Kuzueva, O. G.; Kappe, C. O.; Chupakhin, O. N. J. Fluorine Chem. 2000, 103, 17. http://dx.doi.org/10.1016/S0022-1139(99)00216-X

19. Azizian, J.; Mirza, B.; Mojtahedi, M. M.; Saeed Abaee, M.; Sargordan, M. J. Fluorine Chem. 2008, 129, 1083. http://dx.doi.org/10.1016/j.jfluchem.2008.06.025

20. Burgart, Ya. V.; Kuzueva, O. G.; Pryadeina, M. V.; Kappe, C. O.; Saloutin, V. I. Russ. J. Org. Chem. 2001, 37, 869. http://dx.doi.org/10.1023/A:1012473901354

21. Sabitha, G.; Kumar Reddy, G. S. K.; Reddy, C. S.; Yadav, J. C. Synlett, 2003, 858. http://dx.doi.org/10.1055/s-2003-38734

22. Ryabukhin, S. V.; Plaskon, A. S.; Ostapchuk, E. N.; Volochnyuk, D. M.; Shishkin, O. V.; Tolmachev, A. A. J. Fluorine Chem. 2008, 129, 625. http://dx.doi.org/10.1016/j.jfluchem.2008.05.004

23. Günther, H. NMR Spectroscopy, 2nd Edn.; Wiley: New York, 1995. 
24. Timoshenko, V. M.; Markitanov, Yu. N.; Shermolovich, Yu. G. Chem. Heterocycl. Comp. 2011, 47, 977. http://dx.doi.org/10.1007/s10593-011-0863-4

25. Palanki, M. S. S.; Gayo-Fung, L. M.; Shelvin, G. I., Erdman, P.; Sato, M.; Goldman, M.; Ransone, L. J.; Spooner, C. Bioorg. Med. Chem. Lett. 2002, 12, 2573. http://dx.doi.org/10.1016/S0960-894X(02)00517-6

26. Zanatta, N.; Faoro, D.; Fernandes, Liana da S.; Brondani, P. B.; Flores, D. C.; Flores, A. F. C.; Bonacorso, H. G.; Martins, M. A. P. Eur. J. Org. Chem. 2008, 5832. http://dx.doi.org/10.1002/ejoc.200800822

27. Shaaban, M. R.; Saleh, T. S.; Mayhoub, A. S.; Farag A. M. Eur. J. Med. Chem. 2011, 46, 3690 . http://dx.doi.org/10.1016/j.ejmech.2011.05.033

28. Berber, H.; Soufyane, M.; Mirand, C.; Schmidt, S.; Aubertin, A.-M. Tetrahedron 2001, 57, 7369. http://dx.doi.org/10.1016/S0040-4020(01)00721-9

29. Yu, H.; Han, J.; Chen, J.; Deng, H.; Shao, M.; Zhang, H.; Cao, W. Eur. J. Org. Chem. 2012, 3142 . http://dx.doi.org/10.1002/ejoc. 201200180

30. Sheldrick, G. M. SADABS: Program for scaling and correction of area detector data; University of Göttingen, Germany, 1996.

31. Watkin, D. J.; Prout, C. K.; Carruthers, J. R.; Betteridge, P. W. CRYSTALS; Issue 10. Oxford: Chemical Crystallography Laboratory, University of Oxford, Oxford, 1996.

32. Carruthers, J. R.; Watkin, D. J. Acta Crystallogr. (A) 1979, 35, 698. 